\title{
Bounds for codes and designs in complex subspaces
}

\author{
Aidan Roy
}

Received: 19 July 2008 / Accepted: 11 February 2009 / Published online: 27 February 2009

(C) Springer Science+Business Media, LLC 2009

\begin{abstract}
We introduce the concepts of complex Grassmannian codes and designs. Let $\mathcal{G}_{m, n}$ denote the set of $m$-dimensional subspaces of $\mathbb{C}^{n}$ : then a code is a finite subset of $\mathcal{G}_{m, n}$ in which few distances occur, while a design is a finite subset of $\mathcal{G}_{m, n}$ that polynomially approximates the entire set. Using Delsarte's linear programming techniques, we find upper bounds for the size of a code and lower bounds for the size of a design, and we show that association schemes can occur when the bounds are tight. These results are motivated by the bounds for real subspaces recently found by Bachoc, Bannai, Coulangeon and Nebe, and the bounds generalize those of Delsarte, Goethals and Seidel for codes and designs on the complex unit sphere.
\end{abstract}

Keywords Codes · Designs · Bounds · Grassmannian spaces · Complex subspaces · Linear programming $\cdot$ Delsarte $\cdot$ Association schemes

\section{Introduction}

In this paper, we introduce the concept of complex Grassmannian codes and designs: codes and designs in the collection of fixed-rank subspaces of a complex vector space.

In the 1970's, Delsarte [11] developed a series of excellent bounds for certain error-correcting codes by treating codewords as points in an association scheme and then applying linear programming. Shortly thereafter, Delsarte, Goethals and Seidel [12] showed that the same technique could also be used on systems of points on the real or complex unit sphere, which they called spherical codes and spherical designs; this resulted in important contributions to problems in sphere-packing

A. Roy (凶)

Institute for Quantum Information Science \& Department of Mathematics and Statistics,

University of Calgary, Calgary, Alberta T2N 1N4, Canada

e-mail: aroy@qis.ucalgary.ca 
[10, Chapter 9]. This linear programming technique, which is now known as "Delsarte LP theory", has proved surprisingly portable. Recently, Bachoc, Coulangeon and Nebe [4] and Bachoc, Bannai and Coulangeon [3] generalized the results of Delsarte, Goethals and Seidel to real Grassmannian spaces, and Bachoc [2] pointed out that "the same game" can be played over the complex numbers. In this paper, we investigate more closely the case of complex Grassmannian codes.

The motivation for studying complex Grassmannians comes from the theory of quantum measurements. Roughly speaking, any complex Grassmannian 1-design (or any complex projective 1-design) defines a projective measurement in the theory of quantum mechanics [23, Section 2.2.6]. It has recently been discovered that complex projective 2-designs correspond to quantum measurements that are optimal for the purposes of nonadaptive quantum state tomography [26]. In fact, this is also true in the more general Grassmannian setting: complex Grassmannian 2-designs are the optimal choices of measurements for nonadaptive quantum state tomography when the observer only has access to measurements with a restricted number of outcomes. More details will appear in a paper by Godsil, Rötteler, and the author [15]. Complex Grassmannians also play a role in certain wireless communication protocols [1].

Define $\mathcal{G}_{m, n}$ to be the set of $m$-dimensional subspaces of an $n$-dimensional complex vector space. Without loss of generality, we always assume $m \leq n / 2$. Usually, we represent a subspace $a$ by its $n \times n$ projection matrix $P_{a}$. The inner product on $\mathcal{G}_{m, n}$ is the trace inner product for projection matrices:

$$
\begin{aligned}
\langle a, b\rangle & :=\operatorname{tr}\left(P_{a}^{*} P_{b}\right) \\
& =\operatorname{tr}\left(P_{a} P_{b}\right) .
\end{aligned}
$$

Since $\langle a, b\rangle=\langle b, a\rangle$, the inner product is real. This is a measure of separation, or distance, between two subspaces — note that is not a distance metric per se: the inner product of $P_{a}$ with itself is maximal rather than minimal. However, the chordal distance [9], defined by

$$
d_{c}(a, b):=\sqrt{m-\operatorname{tr}\left(P_{a} P_{b}\right)}
$$

is a monotonic function of the inner product. Given a finite set of inner product values $\mathcal{A}$, an $\mathcal{A}$-code is a subset $S$ of $\mathcal{G}_{m, n}$ such that

$$
\mathcal{A}=\left\{\operatorname{tr}\left(P_{a} P_{b}\right): a, b \in S, a \neq b\right\} .
$$

An $s$-distance set is an $\mathcal{A}$-code with $|\mathcal{A}|=s$. This generalizes the concept of an $s$-distance set on the complex unit sphere: if $u$ and $v$ are unit vectors, then the distance between $u$ and $v$ on the unit sphere is a function of

$$
\left|u^{*} v\right|^{2}=\operatorname{tr}\left(u u^{*} v v^{*}\right) .
$$

We are interested in codes of maximal size for a fixed $\mathcal{A}$ or $s$, and bounds on their size based on zonal polynomials. Table 1 in Section 6 gives a summary of the bounds for small $|\mathcal{A}|$. 
The outline of this paper is as follows. In Section 2, we describe the orbits of pairs of subspaces in $\mathcal{G}_{m, n}$ under the action of $U(n)$ : these orbits play a significant role in the bounds derived later on. In Sections 3, 4 and 5, we develop the necessary representation theory background needed for our LP bounds. In particular, we discuss the decomposition of the square-integrable functions on $\mathcal{G}_{m, n}$ into irreducible representations of $U(n)$, and the zonal polynomials for these representations. The results in this section are all known, and the development is quite similar to that of Bachoc, Coulangeon and Nebe for real Grassmannians. In fact, the complex case is actually easier than the real case, because representations of the unitary group $U(n)$ are easier to describe than representations of the orthogonal group $O(n)$. In Section 6, we develop absolute and relative bounds for $\mathcal{A}$-codes and for a more general type of code called an $f$-code. These bounds for $\mathcal{G}_{m, n}$ reduce to known bounds for complex spherical codes when $m=1$. We compare the bounds to other known bounds for subspaces in Section 7, and in Section 8, we give examples in which the bounds are tight. In Section 9, we consider Grassmannian designs. Complex Grassmannian codes enjoy a form of duality with Grassmannian designs, very similar to real Grassmannian codes or spherical codes. In many cases codes of maximal size or designs of minimal size have the structure of an association scheme, which we describe in Section 10. Finally, in Section 11, we show how a weighted version of a design can be constructed in any dimension.

\section{Orbitals}

In this section we describe the orbits of pairs of elements of $\mathcal{G}_{m, n}$ under the action of $U(n)$.

First, we claim that $\mathcal{G}_{m, n}$ can be identified with a quotient space of the unitary group, $U(n) /(U(m) \times U(n-m))$. For, consider the first $m$ columns of a matrix of $U(n)$ as the basis for a subspace $a$ of dimension $m$ in $\mathbb{C}^{n}$, letting the last $n-m$ columns be a basis for $a^{\perp}$. Then $a$ is invariant under the action of $U(m)$ on the first $m$ columns, while $a^{\perp}$ is invariant under $U(n-m)$.

As a result of this quotient space, $U(n)$ acts on $\mathcal{G}_{m, n}$ as follows: if $U$ is in $U(n)$ and $P_{a}$ is the projection matrix for $a \in \mathcal{G}_{m, n}$, then

$$
U: P_{a} \mapsto U P_{a} U^{*} .
$$

This action is an isometry, in that it preserves the trace inner product on $\mathcal{G}_{m, n}$. Unlike on the complex unit sphere, however, $U(n)$ is not 2-homogeneous on $\mathcal{G}_{m, n}: U(n)$ does not act transitively on pairs of subspaces with the same distance. In other words, the fact that $\operatorname{tr}\left(P_{a} P_{b}\right)=\operatorname{tr}\left(P_{c} P_{d}\right)$ does not imply that there is a unitary matrix mapping $a$ to $c$ and $b$ to $d$. In order to use zonal polynomials, we need to understand the orbits of pairs in $\mathcal{G}_{m, n}$ under this isometry group, which requires principal angles.

Given $a$ and $b$ in $\mathcal{G}_{m, n}$, the principal angles $\theta_{1}, \ldots, \theta_{m}$ between $a$ and $b$ are defined as follows: firstly, $\theta_{1}$ is the smallest angle that occurs between any two unit vectors $a_{1} \in a$ and $b_{1} \in b$ :

$$
\theta_{1}:=\min _{\substack{a_{1} \in a \\ b_{1} \in b}} \arccos \left|a_{1}^{*} b_{1}\right| .
$$


Secondly, $\theta_{2}$ is the smallest angle that occurs between any two unit vectors $a_{2} \in$ $a \cap a_{1}^{\perp}$ and $b_{2} \in b \cap b_{1}^{\perp}$. Similarly define $\theta_{3}, \ldots, \theta_{m}$. These principal angles are closely related to the eigenvalues of $P_{a} P_{b}$ : the first $m$ eigenvalues of $P_{a} P_{b}$ are $\left\{\cos ^{2} \theta_{1}, \ldots, \cos ^{2} \theta_{m}\right\}$. Because of this correspondence, for the remainder of this paper we simply refer to the eigenvalues $y_{i}:=\cos ^{2} \theta_{i}$ (rather than the angles $\theta_{i}$ ) as the principal angles between $a$ and $b$. Note that $n-m$ of the eigenvalues of $P_{a} P_{b}$ are zero, so we need only consider the first $m$ eigenvalues. Conway, Hardin, and Sloane [9] accredit the following lemma to Wong [29, Theorem 2].

Lemma 1 The principal angles characterize the orbits of pairs of subspaces un$\operatorname{der} U(n)$.

Proof Suppose $U \in U(n)$ maps projection matrices $P_{a}$ and $P_{b}$ to $P_{c}$ and $P_{d}$ respectively. Then by similarity, the eigenvalues of

$$
P_{c} P_{d}=\left(U P_{a} U^{*}\right)\left(U P_{b} U^{*}\right)=U P_{a} P_{b} U^{*}
$$

are the same as the eigenvalues of $P_{a} P_{b}$.

Conversely, we show that if $P_{a} P_{b}$ and $P_{c} P_{d}$ have the same eigenvalues, then some unitary matrix $U$ maps $a$ to $c$ and $b$ to $d$. We do this by unitarily mapping $a$ and $b$ into a canonical form that depends only on the eigenvalues of $P_{a} P_{b}$.

Let $M_{a}$ be an $n \times m$ matrix whose columns $\left[a_{1}, \ldots, a_{m}\right]$ are an orthonormal basis for $a$, so that $M_{a} M_{a}^{*}=P_{a}$ and $M_{a}^{*} M_{a}=I$. Similarly define $M_{b}=\left[b_{1}, \ldots, b_{m}\right]$ for $b$. Suppose $M_{a}^{*} M_{b}$ has singular value decomposition $U D V^{*}$, where $U$ and $V$ are $m \times m$ unitary and $D$ is $m \times m$ diagonal. Then $\left(M_{a} U\right)^{*}\left(M_{b} V\right)=D$. Since the columns of $M_{a} U$ are another orthonormal basis for $a$, without loss of generality we replace $M_{a}$ by $M_{a} U$ and likewise replace $M_{b}$ with $M_{b} V$. In other words, we may assume without loss of generality that $M_{a}^{*} M_{b}=D$, where $D$ is a diagonal matrix of singular values.

Next, define the columns of $N_{a}=\left[a_{m+1}, \ldots, a_{n}\right]$ to be any orthonormal basis for $a^{\perp}$, so that $N_{a} N_{a}^{*}=I-P_{a}$ and $N_{a}^{*} N_{a}=I$. Further assume that $N_{a}^{*} M_{b}=Q R$, where $Q$ is $(n-m) \times(n-m)$ unitary and $R$ is $(n-m) \times m$ upper triangular (the $Q R$-decomposition of $N_{a}^{*} M_{b}$ ). Then $Q^{*} N_{a}^{*} M_{b}=R$, and the columns of $N_{a} Q$ form another orthonormal basis for $a^{\perp}$. Replacing $N_{a}$ by $N_{a} Q$, we may assume without loss of generality that $N_{a}^{*} M_{b}$ is upper triangular.

Finally, let $U_{a}:=\left(\begin{array}{c}M_{a}^{*} \\ N_{a}^{*}\end{array}\right)$; this is an $n \times n$ unitary matrix. Then

$$
U_{a} M_{a}=\left(\begin{array}{c}
I_{m} \\
0
\end{array}\right) ; \quad U_{a} M_{b}=\left(\begin{array}{l}
D \\
R
\end{array}\right)
$$

If $P_{a} P_{b}$ has eigenvalues $\cos ^{2} \theta_{i}$, then $M_{a}^{*} M_{b}=D$ has singular values $\cos \theta_{i}$. Moreover, since $U_{a} M_{b}$ has orthonormal columns, it follows that $R$ also has orthogonal columns. We may therefore assume that $R$ is not just upper triangular but diagonal, with diagonal entries $\sin \theta_{i}$. Thus $U_{a}$ is a unitary matrix which maps $M_{a}$ and $M_{b}$ into 
the form

$$
M_{a} \mapsto\left(\begin{array}{c}
I_{m} \\
0
\end{array}\right), \quad M_{b} \mapsto\left(\begin{array}{ccc}
\cos \theta_{1} & & \\
& \ddots & \\
& & \cos \theta_{m} \\
\sin \theta_{1} & & \\
& \ddots & \\
& & \sin \theta_{m} \\
& 0 &
\end{array}\right) .
$$

Since any pair $\left(M_{a}, M_{b}\right)$ with principal angles $\cos ^{2} \theta_{i}$ can be mapped to this canonical form, it follows that the eigenvalues of $P_{a} P_{b}$ characterize the orbits of pairs $(a, b)$ under the unitary group.

\section{Representations}

In this section and the next, we develop the representation theory needed for Grassmannian LP bounds.

Because $U(n)$ is a compact Lie group, up to normalization it has a unique invariant measure (the Haar measure). Since $U(n)$ acts transitively on $\mathcal{G}_{m, n}$, this induces a unique invariant measure on $\mathcal{G}_{m, n}$, which we normalize so that

$$
\int_{\mathcal{G}_{m, n}} d a=1
$$

With this measure, the square-integrable functions $L^{2}\left(\mathcal{G}_{m, n}\right)$ are those functions $f$ : $\mathcal{G}_{m, n} \rightarrow \mathbb{C}$ such that $\int|f(a)|^{2} d a$ is finite. As is standard for compact Lie groups, we work with square-integrable functions to find irreducible representations. The group $U(n)$ acts on $f \in L^{2}\left(\mathcal{G}_{m, n}\right)$ as follows:

$$
(U f)\left(P_{a}\right):=f\left(U^{*} P_{a} U\right)
$$

where $P_{a}$ is the projection matrix for $a \in \mathcal{G}_{m, n}$. It follows that $L^{2}\left(\mathcal{G}_{m, n}\right)$ is a representation of $U(n)$. There is a natural inner product on this space:

$$
\langle f, g\rangle:=\int_{\mathcal{G}_{m, n}} \overline{f(a)} g(a) d a .
$$

Equivalently, we may write

$$
\langle f, g\rangle:=\int_{U(n)} \overline{f\left(U^{*} P_{a} U\right)} g\left(U^{*} P_{a} U\right) d U,
$$

where $d U$ is the Haar measure on $U(n)$ and $P_{a}$ is some fixed projection matrix. As we will see, this representation can be decomposed into orthogonal, irreducible subrepresentations, and the decomposition is multiplicity-free: no irreducible representation of $U(n)$ occurs more than once in $L^{2}\left(\mathcal{G}_{m, n}\right)$. 
Since $U(n)$ is a compact Lie group, its irreducible representations are wellstudied: see for example [7, 13, 17, 27]. Every irreducible representation is indexed by a dominant weight [27, Theorem 7.34]. In the case of $U(n)$, we may take these weights to have the form [7, Theorem 38.3]

$$
\lambda=\left(\lambda_{1}, \ldots, \lambda_{n}\right): \lambda_{1} \geq \lambda_{2} \geq \cdots \geq \lambda_{n}, \lambda_{i} \in \mathbb{Z}
$$

The dimension of the irreducible representation $V_{\lambda}$ indexed by $\lambda$ is given by Weyl's character formula [27, Theorem 7.32]. In the case of $U(n)$, the formula reduces to:

$$
\operatorname{dim} V_{\lambda}=\prod_{1 \leq i<j \leq n} \frac{\lambda_{i}-\lambda_{j}+j-i}{j-i}
$$

For example, the standard representation of $U(n)$ is indexed by $\lambda=(1,0, \ldots, 0)$, which gives

$$
\operatorname{dim} V_{(1,0, \ldots, 0)}=n \text {. }
$$

Note that there is more than one irreducible representation with the same dimension.

Each dominant weight may also be thought of as a form acting on a maximal torus of the Lie group. Here $\lambda$ acts on the diagonal matrix $d=\operatorname{diag}\left(d_{1}, \ldots, d_{n}\right) \in U(n)$ as follows:

$$
d^{\lambda}:=\prod_{i=1}^{n} d_{i}^{\lambda_{i}} .
$$

The next section describes exactly which of these forms contribute to the decomposition of $L^{2}\left(\mathcal{G}_{m, n}\right)$.

\section{Symmetric spaces}

The space $U(n) / U(m) \times U(n-m)$ is an example of a symmetric space: a quotient space $G / K$ such that $G$ is a connected semisimple Lie group and $K$ is the fixed point set of an involutive automorphism of $G$. In this section, we use results from Goodman and Wallach [17] to explain how the decomposition of representations of $\mathcal{G}_{m, n}$ follows from this structure.

Let $s_{m}$ denote the $m \times m$ matrix with backwards diagonal entries of 1 and 0 elsewhere:

$$
s_{m}:=\left(\begin{array}{lll}
0 & & 1 \\
& . & \\
1 & & 0
\end{array}\right) .
$$

Also define

$$
J_{m, n}:=\left(\begin{array}{ccc} 
& & s_{m} \\
& I_{n-2 m} & \\
s_{m} & &
\end{array}\right)
$$


and consider the involution $\theta(M):=J_{m, n} M J_{m, n}$ on $G L_{n}(\mathbb{C})$. The fixed points of $\theta$ have the form

$$
M=\left(\begin{array}{ccc}
a & b & c \\
d & e & d s_{m} \\
s_{m} c s_{m} & s_{m} b & s_{m} a s_{m}
\end{array}\right),
$$

so the fixed point set in $G L_{n}(\mathbb{C})$ is isomorphic to $G L_{m}(\mathbb{C}) \times G L_{n-m}(\mathbb{C})$.

Lemma 2 The fixed point set $K$ of $\theta$ in $G=U(n)$ is isomorphic to $U(m) \times U(n-m)$. Therefore $\mathcal{G}_{m, n}$ is a symmetric space.

Proof For $a=\left(a_{1}, \ldots, a_{m}\right)$, let $\breve{a}$ denote the reversal of $a$, namely

$$
\breve{a}:=s_{m} a=\left(a_{m}, \ldots, a_{1}\right) .
$$

If $a, b$, and $c$ have length $m, n-2 m$ and $m$ respectively, then we have $J_{m, n}(a, b, c)^{T}=$ $(\breve{c}, b, \breve{a})^{T}$. Therefore the 1 and -1 eigenspaces of $J_{m, n}$ are $V_{+}=\{(a, b, \breve{a})\}$ and $V_{-}=\{(a, 0,-\breve{a})\}$ respectively. These spaces are orthogonal with respect to the form $(x, y) \mapsto x^{*} y$.

Now $K$ is the set of points in $U(n)$ which commute with $J_{m, n}$. So decomposing $\mathbb{C}^{n}$ into $V_{+} \oplus V_{-}$, we have that $K$ is the set of points in $U(n)$ which leave both $V_{+}$ and $V_{-}$invariant. In other words, $K$ is the set of points which preserve the form $(x, y) \mapsto x^{*} y$ on the subspaces $V_{+}$and $V_{-}$. Thus

$$
K \cong U\left(V_{+}\right) \times U\left(V_{-}\right) \cong U(n-m) \times U(m) \text {. }
$$

The fact that $K$ is the fixed point set of $\theta$ in $G$ implies ([17, Theorem 12.3.5]) that $(G, K)$ is a spherical pair: for every irreducible representation $V_{\lambda}$ of $G$, the subspace $V_{\lambda}^{K}$ of points fixed by $K$ satisfies $\operatorname{dim} V_{\lambda}^{K} \leq 1$. Those representations such that $V_{\lambda}^{K}$ has dimension exactly 1 are called spherical representations. The following theorem [18, Theorem V.4.3] explains how those representation relate to $L^{2}(G / K)$.

Theorem 1 Let $G$ be a compact simply connected semisimple Lie group, and let $K \leq G$ be the fixed point group of an involutive automorphism of $G$. Further let $\hat{G}_{K}$ denote the set of equivalence classes of spherical representations $V_{\lambda}$ of $G$ with respect to $K$. Then $L^{2}(G / K)$ is a multiplicity-free representation of $G$, and

$$
L^{2}(G / K) \cong \bigoplus_{\lambda \in \hat{G}_{K}} V_{\lambda}
$$

To describe which representations are spherical, we now consider diagonal subgroups of $G$ and $K$. For $d=\left(d_{1}, \ldots, d_{n}\right)$, let $\operatorname{diag}(d)$ denote the diagonal matrix with diagonal entries $d_{1}, \ldots, d_{n}$. Firstly, note that $\operatorname{diag}(d)$ is in $U(n)$ if and only if $\left|d_{i}\right|=1$ for all $i$. Secondly, note that if $d=\operatorname{diag}(a, b, c)$ with $a$ and $c$ of length $m$, then $\theta(d)=(\breve{c}, b, \breve{a})$. It follows that the diagonal group

$$
T:=\left\{\operatorname{diag}\left(a_{1}, \ldots, a_{m}, b_{m+1}, \ldots, b_{n-m}, a_{m}, \ldots, a_{1}\right):\left|a_{i}\right|=\left|b_{i}\right|=1\right\}
$$


is contained in $K$. In fact, it is a maximal subgroup of $K$ isomorphic to $(\mathbb{R} / \mathbb{Z})^{r}$ : this is called a maximal torus of $K$.

Recall that the irreducible representations of $G$ are indexed by the dominant weights $\lambda=\left(\lambda_{1}, \ldots, \lambda_{n}\right)$, where $\lambda_{i} \geq \lambda_{i+1}$ and $\lambda_{i} \in \mathbb{Z}$. Now the spherical representations of $G$ with respect to $K$ are indexed by those particular dominant weights such that $t^{\lambda}=1$ for all $t=\left(t_{1}, \ldots, t_{n}\right)$ in the torus $T$ (see Goodman and Wallach [17, p. 540]). So a dominant weight $\lambda$ is spherical if it has the form

$$
\lambda=\left(\lambda_{1}, \ldots, \lambda_{m}, 0, \ldots, 0,-\lambda_{m}, \ldots,-\lambda_{1}\right)
$$

with $\lambda_{1} \geq \cdots \geq \lambda_{m} \geq 0$ and $\lambda_{i} \in \mathbb{Z}$. In other words:

Theorem 2 The irreducible representations of $U(n)$ occurring in $L^{2}\left(\mathcal{G}_{m, n}\right)$ are in one-to-one correspondence with the integer partitions with at most $m$ parts.

For any partition $\mu$, we let $H_{\mu}(n)$, or simply $H_{\mu}$, denote the irreducible representation in $L^{2}\left(\mathcal{G}_{m, n}\right)$ isomorphic to $V_{(\mu, 0, \ldots, 0,-\breve{\mu})}$. The Weyl character formula (equation (1)) now tells us the dimension of each $H_{\mu}$. The first few dimensions are:

$$
\begin{aligned}
\operatorname{dim} H_{(0)}=\operatorname{dim} V_{(0, \ldots, 0)} & =1 \\
\operatorname{dim} H_{(1)}=\operatorname{dim} V_{(1,0, \ldots, 0,-1)} & =n^{2}-1 \\
\operatorname{dim} H_{(2)} & =\frac{n^{2}(n-1)(n+3)}{4} \\
\operatorname{dim} H_{(1,1)} & =\frac{n^{2}(n+1)(n-3)}{4} \\
\operatorname{dim} H_{(2,1)} & =\frac{\left(n^{2}-1\right)^{2}\left(n^{2}-9\right)}{9} \\
\operatorname{dim} H_{(k)} & =\left(\begin{array}{c}
n+k-2 \\
k
\end{array}\right)^{2} \frac{n+2 k-1}{n-1} \\
\operatorname{dim} H_{(1, \ldots, 1)} & =\left(\begin{array}{c}
n+1 \\
k
\end{array}\right)^{2} \frac{n-2 k+1}{n+1}
\end{aligned}
$$

If $m=1$, then $\mathcal{G}_{m, n}$ is the complex projective space $\mathbb{C} P^{n-1}$, and only the spaces $H_{(k)}$ occur. In that case $H_{(k)}$ is isomorphic to the space $\operatorname{Harm}(k, k)$ of harmonic polynomials of homogeneous degree $k$ in both $z$ and $\bar{z}$, where $z=\left(z_{1}, \ldots, z_{n}\right)$ is a point on the unit sphere in $\mathbb{C}^{n}$. Those harmonic polynomials were used by Delsarte, Goethals, and Seidel in their LP bounds for codes and designs on the complex unit sphere [12].

We now record another representation of $U(n)$ in $L^{2}\left(\mathcal{G}_{m, n}\right)$ that we need for our bounds on codes and designs. Given an nonincreasing sequence of nonnegative integers $\mu=\left(\mu_{1}, \mu_{2}, \ldots\right)$, we say $\mu$ has size $k$ and write $|\mu|=k$ if $\mu$ is a partition of $k$; that is, $\sum_{i} \mu_{i}=k$. We also say $\mu$ has length $l$ and write $\operatorname{len}(\mu)=l$ if $\mu$ has $l$ nonzero entries. For example, $(2,1,0, \ldots)$ has size 3 and length 2 . Then for fixed $\mathcal{G}_{m, n}$, define 
$H_{k}=H_{k}(m, n)$ as follows:

$$
H_{k}(m, n):=\bigoplus_{\substack{|\mu| \leq k \\ \operatorname{len}(\mu) \leq m}} H_{\mu}(n) .
$$

The space $H_{0}$ has dimension 1 and consists of the constant functions on $\mathcal{G}_{m, n}$. For $k>0$, the representation $H_{k}$ is reducible, and $H_{k-1}$ is contained in $H_{k}$. When $m=1$, $H_{k}$ is isomorphic to the space of homogeneous polynomials degree $k$ in both $z$ and $\bar{z}$ on the unit sphere in $\mathbb{C}^{n}$. In the next section, we will see that $H_{k}$ is also the span of the symmetric polynomials of degree at most $k$ on the principal angles between $a$ and $b$ in $\mathcal{G}_{m, n}$, for fixed $a$. Moreover, if $g$ and $h$ are polynomials in $H_{k}$ and $H_{k^{\prime}}$ respectively, then $g h$ is in $H_{k+k^{\prime}}$, and in fact $H_{k+k^{\prime}}$ is spanned by polynomials of that form.

We will also see in the next section that $H_{k}$ is the space of polynomials $f(b)$ which are homogeneous of degree $k$ in the entries of $P_{b}$, the projection matrix of $b \in \mathcal{G}_{m, n}$. It follows that for fixed $a \in \mathcal{G}_{m, n}$, the inner product function $b \mapsto\langle a, b\rangle=\operatorname{tr}\left(P_{a} P_{b}\right)$ is in $H_{1}(n)$.

James and Constantine [20] further investigated the irreducible subspaces of $L^{2}\left(\mathcal{G}_{m, n}\right)$, finding zonal polynomials for each irreducible representation. We describe those results in the following section.

\section{Zonal polynomials}

A zonal polynomial at a point $a \in \mathcal{G}_{m, n}$ is a function on points $b \in \mathcal{G}_{m, n}$ which depends only on the principal angles between $a$ and $b$. Given a symmetric polynomial $f\left(x_{1}, \ldots, x_{m}\right) \in \mathbb{R}\left[x_{1}, \ldots, x_{m}\right]$ of degree $k$, we define the zonal polynomial of $f$ at $a$ as follows: if $y(a, b)=\left(y_{1}, \ldots, y_{m}\right)$ are the principal angles of $a$ and $b$, then

$$
f_{a}(b):=f\left(y_{1}, \ldots, y_{m}\right) .
$$

Since $f$ is a symmetric polynomial of degree at most $k$ in the principal angles, it is in $H_{k}(m, n)$. If $P_{a}$ and $P_{b}$ are the projection matrices for $a$ and $b$, then $b \mapsto \operatorname{tr}\left(P_{a} P_{b}\right)$ is an example of a zonal polynomial, since $\operatorname{tr}\left(P_{a} P_{b}\right)=\sum_{i=1}^{m} y_{i}$, a symmetric polynomial of degree 1.

There is a particular set of zonal polynomials that play a special role in the theory of Delsarte bounds. Let $H_{\mu}$ be an irreducible representation in $L^{2}\left(\mathcal{G}_{m, n}\right)$. Then for each $a \in \mathcal{G}_{m, n}$, define the zonal orthogonal polynomial or zonal spherical polynomial $Z_{\mu, a}$ to be the unique element of $H_{\mu}$ such that for every $p \in H_{\mu}$,

$$
\left\langle Z_{\mu, a}, p\right\rangle=p(a) .
$$

From equation (2) it follows that the set $\left\{Z_{\mu}, a: a \in \mathcal{G}_{m, n}\right\}$ spans $H_{\mu}$. These zonal polynomials are invariant under the unitary group, in the following sense:

$$
Z_{\mu, b}(a)=\left\langle U^{*} Z_{\mu, a}, U^{*} Z_{\mu, b}\right\rangle=\left\langle Z_{\mu, U a}, Z_{\mu, U b}\right\rangle=Z_{\mu, U b}(U a) .
$$

(By $U a$ we mean the action $U: P_{a} \mapsto U P_{a} U^{*}$.) The value of $Z_{\mu, b}(a)$ depends on the $U(n)$-orbit of $(a, b)$ and therefore depends on the principal angles of $a$ and $b$. With 
this in mind we sometimes write $Z_{\mu, a}(b)=Z_{\mu}(a, b)$ or $Z_{\mu, a}(b)=Z_{\mu}\left(y_{1}, \ldots, y_{m}\right)$, where $\left(y_{1}, \ldots, y_{m}\right)$ are the principal angles of $a$ and $b$.

Schur orthogonality [27, Theorem 3.3] for irreducible representations implies that $Z_{\mu, a}$ and $Z_{\nu, b}$ are orthogonal for $\mu \neq v$. So, we have

$$
\left\langle Z_{\mu, a}, Z_{v, b}\right\rangle=\delta_{\mu, v} Z_{\mu}(a, b) .
$$

Moreover, $Z_{\mu, a}(b)=Z_{\mu, b}(a)$ is in fact real and symmetric in $a$ and $b$. The zonal polynomials satisfy some other important properties, including the following positivity condition:

Lemma 3 For any subset $S \subseteq \mathcal{G}_{m, n}$,

$$
\sum_{a, b \in S} Z_{\mu}(a, b) \geq 0 .
$$

Equality holds if and only if $\sum_{a \in S} Z_{\mu, a}=0$.

Proof We have

$$
\begin{aligned}
\sum_{a, b \in S} Z_{\mu}(a, b) & =\sum_{a, b \in S}\left\langle Z_{\mu, a}, Z_{\mu, b}\right\rangle \\
& =\left\langle\sum_{a \in S} Z_{\mu, a}, \sum_{b \in S} Z_{\mu, b}\right\rangle \\
& \geq 0 .
\end{aligned}
$$

Equality holds only when $\sum_{a \in S} Z_{\mu, a}=0$.

The second important condition the zonal polynomials satisfy is called the addition formula:

Lemma 4 Let $e_{1}, \ldots, e_{N}$ be an orthonormal basis for the irreducible subspace $H_{\mu}$. Then

$$
\sum_{i=1}^{N} \overline{e_{i}(a)} e_{i}(b)=Z_{\mu}(a, b) .
$$

Proof Since $Z_{\mu, a}$ is in $H_{\mu}$, we may write it as a linear combination of $e_{1}, \ldots, e_{N}$ :

$$
\begin{aligned}
Z_{\mu, a} & =\sum_{i=1}^{N}\left\langle e_{i}, Z_{\mu, a}\right\rangle e_{i} \\
& =\sum_{i} \overline{e_{i}(a)} e_{i} .
\end{aligned}
$$

So, it follows that $Z_{\mu, a}(b)=\sum_{i} \overline{e_{i}(a)} e_{i}(b)$. 
James and Constantine give an explicit formula for the zonal orthogonal polynomials of $\mathcal{G}_{m, n}$ in terms of Schur polynomials, the irreducible characters of $S L(m, \mathbb{C})$. If $y=\left(y_{1}, \ldots, y_{m}\right)$ are variables and $\sigma=\left(s_{1}, \ldots, s_{m}\right)$ is an integer partition into at most $m$ parts, then the (unnormalized) Schur polynomial is defined as

$$
X_{\sigma}(y):=\frac{\operatorname{det}\left(y_{i}^{s_{j}+m-j}\right)_{i, j}}{\operatorname{det}\left(y_{i}^{k-j}\right)_{i, j}} .
$$

Each Schur polynomial is a symmetric polynomial in $\left(y_{1}, \ldots, y_{m}\right)$. For more information about Schur polynomials, see Stanley [28, Chapter 7]. The normalized Schur polynomial $X_{\sigma}^{*}$ is the multiple of $X_{\sigma}$ such that $X_{\sigma}^{*}(1, \ldots, 1)=1$.

To define the zonal orthogonal polynomials for $\mathcal{G}_{m, n}$, first define the ascending product

$$
(a)_{s}:=a(a+1) \cdots(a+s-1),
$$

and given a partition $\sigma=\left(s_{1}, \ldots, s_{m}\right)$, define complex hypergeometric coefficients

$$
[a]_{\sigma}:=\prod_{i=1}^{m}(a-i+1)_{s_{i}} .
$$

Further assume we have a partial order $\leq$ on partitions defined such that $\left(s_{1}, \ldots, s_{m}\right) \leq\left(k_{1}, \ldots, k_{l}\right)$ if and only if $s_{i} \leq k_{i}$ for all $i$. Letting $y+1:=\left(y_{1}+\right.$ $\left.1, \ldots, y_{m}+1\right)$, the complex hypergeometric binomial coefficients $\left[\begin{array}{c}\kappa \\ \sigma\end{array}\right]$ are given by the formula

$$
X_{\kappa}^{*}(y+1)=\sum_{\sigma \leq \kappa}\left[\begin{array}{l}
\kappa \\
\sigma
\end{array}\right] X_{\sigma}^{*}(y) .
$$

We can now define the zonal orthogonal polynomials for $\mathcal{G}_{m, n}$. The following result is due to James and Constantine [20].

Theorem 3 Let

$$
\rho_{\sigma}:=\sum_{i=1}^{m} s_{i}\left(s_{i}-2 i+1\right)
$$

and let $\sigma$ and $\kappa$ partition $s$ and $k$ respectively. Also let

$$
[c]_{(\kappa, \sigma)}:=\sum_{i} \frac{\left[\begin{array}{c}
\kappa \\
\sigma_{i}
\end{array}\right]\left[\begin{array}{c}
\sigma_{i} \\
\sigma
\end{array}\right]}{(k-s)\left[\begin{array}{l}
\kappa \\
\sigma
\end{array}\right]} \frac{[c]_{\left(\kappa, \sigma_{i}\right)}}{\left(c+\frac{\rho_{\kappa}-\rho_{\sigma}}{k-s}\right)},
$$

where the summation is over partitions $\sigma_{i}=\left(s_{1}, \ldots, s_{i-1}, s_{i}+1, s_{i+1}, \ldots\right)$ that are nonincreasing. Then up to normalization, the zonal orthogonal polynomial for $H_{\kappa}$ is

$$
Z_{\kappa}(y):=\sum_{\sigma \leq \kappa} \frac{(-1)^{s}\left[\begin{array}{l}
\kappa \\
\sigma
\end{array}\right]_{[c]_{(\kappa, \sigma)}}}{[a]_{\sigma}} X_{\sigma}^{*}(y),
$$


where $y=\left(y_{1}, \ldots, y_{m}\right)$ is the set of principal angles.

The first few normalized Schur polynomials are:

$$
\begin{aligned}
X_{(0)}^{*}(y) & =1 \\
X_{(1)}^{*}(y) & =\frac{1}{m} \sum_{i=1}^{m} y_{i} \\
X_{(1,1)}^{*}(y) & =\frac{1}{\left(\begin{array}{c}
m \\
2
\end{array}\right)} \sum_{i<j} y_{i} y_{j} \\
X_{(2)}^{*}(y) & =\frac{1}{\left(\begin{array}{c}
m+1 \\
2
\end{array}\right)}\left(\sum_{i=1}^{m} y_{i}^{2}+\sum_{i<j} y_{i} y_{j}\right) .
\end{aligned}
$$

Up to normalization by a constant, the first few zonal orthogonal polynomials are:

$$
\begin{aligned}
Z_{(0)}(y) & =1 \\
Z_{(1)}(y) & =n X_{(1)}^{*}(y)-m \\
Z_{(1,1)}(y) & =(n-1)(n-2) X_{(1,1)}^{*}(y)-2(n-1)(m-1) X_{(1)}^{*}(y)+m(m-1) \\
Z_{(2)}(y) & =(n+1)(n+2) X_{(2)}^{*}(y)-2(n+1)(m+1) X_{(1)}^{*}(y)+m(m+1) .
\end{aligned}
$$

The correct normalizations satisfy

$$
\left\langle Z_{\mu, a}, Z_{\mu, a}\right\rangle=Z_{\mu}(1,1, \ldots, 1)=\operatorname{dim} H_{\mu} .
$$

With the exception of the case $\mu=(0)$ (which is normalized correctly in the formula above), normalizations for $Z_{\mu}$ do not play a role in the results which follow.

From the result of James and Constantine, a few observations are apparent. Firstly, the zonal orthogonal polynomials $Z_{\mu}(y)$, like the Schur polynomials, are symmetric polynomials in $y_{1}, \ldots, y_{m}$, and the polynomials with $|\mu| \leq k$ form an orthonormal basis for the symmetric polynomials in $y_{1}, \ldots, y_{m}$ of degree at most $k$. Secondly, we have the following useful description of $H_{k}$.

Lemma $5 H_{k}(m, n)$ is the space of polynomials $\mathcal{G}_{m, n} \rightarrow \mathbb{C}$ which are homogeneous of degree $k$ in the entries of the projection matrices for the subspaces.

Proof For convenience, let $\operatorname{Hom}_{k}$ denote the space of polynomials $f(b)$ which are homogeneous of degree $k$ in the entries of $P_{b}$, for $b \in \mathcal{G}_{m, n}$. First, we claim that $H_{k}$ is contained in $\operatorname{Hom}_{k}$. Since the zonal polynomials $\left\{Z_{\mu, a}: a \in \mathcal{G}_{m, n}\right\}$ span $H_{\mu}$, the zonal polynomials $\left\{Z_{\mu, a}:|\mu| \leq k, a \in \mathcal{G}_{m, n}\right\}$ span $H_{k}$, and it suffices to show that $Z_{\mu, a}$ is in $\operatorname{Hom}_{k}$. But $Z_{\mu, a}(b)$ is a symmetric polynomial of the principal angles $y(a, b)$, which are precisely the nonzero eigenvalues of $P_{a} P_{b}$. Moreover, a standard theorem from linear algebra [19, Theorem 1.2.12] states that the $j$-th elementary symmetric function of the eigenvalues of a matrix is the sum of all $j \times j$ principal minors. 
Therefore every symmetric polynomial of degree $k$ in the eigenvalues of $P_{a} P_{b}$ is also a homogeneous polynomial of degree $k$ in the entries of $P_{a} P_{b}$, which is in turn a homogeneous polynomial of degree $k$ in the entries of $P_{b}$.

Next we claim that $\operatorname{Hom}_{k}$ and $H_{k}$ are actually equal. To see this, consider the zonal polynomials in $\mathrm{Hom}_{k}$ : the degree- $k$ polynomials $f_{a}(b)$ in the entries of $P_{b}$ which depend only on the $U(n)$-orbit of $(a, b)$. These zonal polynomials are symmetric functions of the principal angles $y(a, b)=y_{1}, \ldots, y_{m}$, which depend only on the projection of a basis of $b$ onto the subspace $a$. Therefore, it suffices to consider degree- $k$ polynomials in the entries of $P_{a} P_{b} P_{a}$ (which are also degree- $k$ polynomials in the entries of $P_{b}$ ). Now choose the unitary matrix $U_{a}$ in the proof of Lemma 1 so that

$$
U_{a} P_{a} U_{a}^{*}=\operatorname{diag}(1, \ldots, 1,0, \ldots, 0), \quad U_{a} P_{a} P_{b} P_{a} U_{a}^{*}=\operatorname{diag}\left(y_{1}, \ldots, y_{m}, 0, \ldots, 0\right) .
$$

Since the zonal polynomials are symmetric functions of $y_{1}, \ldots, y_{m}$ and polynomials of degree $k$ in the entries of $U P_{a} P_{b} P_{a} U^{*}$, they are symmetric polynomials of degree $k$ in $y_{1}, \ldots, y_{m}$. Thus every zonal polynomial of $\mathrm{Hom}_{k}$ is also a zonal polynomial of $H_{k}$. Since the two spaces have the same zonal polynomials, and the zonal polynomials span the entire space, the two spaces are equal.

\section{Bounds}

Recall that an $\mathcal{A}$-code is a collection $S$ of subspaces in $\mathcal{G}_{m, n}$ such that $\langle a, b\rangle=$ $\operatorname{tr}\left(P_{a} P_{b}\right) \in \mathcal{A}$ for every $a \neq b$ in $S$. In this section, we find upper bounds on the size of an $\mathcal{A}$-code in terms of either the cardinality of $\mathcal{A}$ or the elements of $\mathcal{A}$. A summary of the results for $|\mathcal{A}| \leq 2$ is given in Table 1 .

Table 1 Upper bounds on $|S|$, when $S \subseteq \mathcal{G}_{m, n}$ is an $\mathcal{A}$-code

\begin{tabular}{|l||c|c|}
\hline \multicolumn{1}{|c|}{$\mathcal{A}$} & $\{\alpha\}$ & $\{\alpha, \beta\}$ \\
\hline $\begin{array}{l}\text { Absolute } \\
\text { bound }\end{array}$ & $n^{2}$ & $\left(\begin{array}{c}n^{2} \\
2\end{array}\right) \quad(m>1)$ \\
\hline $\begin{array}{l}\text { Relative } \\
\text { bound }\end{array}$ & $\frac{n(m-\alpha)}{m^{2}-n \alpha}$ & $\frac{n(m-\alpha)(m-\beta)}{m^{2}\left[\frac{(m+1)^{2}}{2(n+1)}+\frac{(m-1)^{2}}{2(n-1)}-(\alpha+\beta)+\frac{n \alpha \beta}{m^{2}}\right]}$ \\
& $\alpha<\frac{m^{2}}{n}$ & $\alpha+\beta \leq \frac{2\left(m^{2} n-4 m+n\right)}{n^{2}-4}$, \\
$\begin{array}{l}\text { Relative } \\
\text { bound } \\
\text { conditions }\end{array}$ & $\alpha+\beta-\frac{n \alpha \beta}{m^{2}}<\frac{m^{2} n-2 m+n}{n^{2}-1}$ \\
\hline
\end{tabular}


If $\mathcal{A}=\left\{\alpha_{1}, \ldots, \alpha_{k}\right\}$, then the annihilator of $\mathcal{A}$ is the symmetric function

$$
\operatorname{ann}_{\mathcal{A}}(y):=\prod_{i=1}^{k}\left(\sum_{j=1}^{m} y_{j}-\alpha_{i}\right) .
$$

The significance of the annihilator is that if $S$ is an $\mathcal{A}$-code, then $\operatorname{ann}_{\mathcal{A}}(y(a, b))=0$ for any $a \neq b$ in $S$. More generally, given any symmetric polynomial $f$ satisfying $f(1,1, \ldots, 1) \neq 0$, an $f$-code is a collection $S$ of subspaces such that for every $a \neq b$ in $S$, the principal angles $y(a, b)=\left(y_{1}, \ldots, y_{m}\right)$ satisfy $f\left(y_{1}, \ldots, y_{m}\right)=0$. If $\mathcal{A}$ is any set of inner product values and $f$ is the annihilator of $\mathcal{A}$, then an $\mathcal{A}$-code is also an $f$-code.

Theorem 4 If $S \subseteq \mathcal{G}_{m, n}$ is an $f$-code, with $\operatorname{deg}(f)=k$, then

$$
|S| \leq \operatorname{dim}\left(H_{k}(m, n)\right)
$$

In particular, if $S$ is a $k$-distance set, then the annihilator of the code has degree $k$, so $|S| \leq \operatorname{dim}\left(H_{k}(m, n)\right)$. Note that since $H_{k}(m, n)$ is the space of homogeneous polynomials of degree $k$ in the $n \times n$ projection matrices for $\mathcal{G}_{m, n}$, we have

$$
\operatorname{dim}\left(H_{k}(m, n)\right) \leq\left(\begin{array}{c}
n^{2}+k-1 \\
k
\end{array}\right) .
$$

Proof If $S$ is an $f$-code, consider the zonal polynomials $f_{a}(b):=f(y(a, b))$, for $a \in S$. Since $f_{a}(b)$ is a degree- $k$ symmetric polynomial in $y(a, b)$, it is an element of $H_{k}(m, n)$. Since $f_{a}(b)=0$ for every $b \in S$ except $a$, and $f_{a}(a) \neq 0$, the set $\left\{f_{a}: a \in S\right\}$ is linearly independent. Thus the number of functions $|S|$ is at most the dimension of the space $H_{k}(m, n)$.

If equality holds, then the functions $f_{a}$ form a basis for the space. Moreover, the space $H_{k}(m, n)$ is exactly the space of functions on $S$.

Corollary 1 If $S$ is a 1-distance set in $\mathcal{G}_{m, n}$, then

$$
|S| \leq n^{2} \text {. }
$$

If $S$ is a 2-distance set in $\mathcal{G}_{m, n}(m>1)$, then

$$
|S| \leq\left(\begin{array}{c}
n^{2} \\
2
\end{array}\right)
$$

Proof Use Theorem 4 together with the facts that $\operatorname{dim}\left(H_{1}(m, n)\right)=n^{2}$ and for $m>1$, $\operatorname{dim}\left(H_{2}(m, n)\right)=\left(\begin{array}{c}n^{2} \\ 2\end{array}\right)$.

Theorem 4 is called the absolute bound for Grassmannian codes, because the bound depends only on the number of different distances that occur in $S$. It is the 
complex analogue of the bound for real Grassmannian spaces given by Bachoc, Bannai and Coulangeon [3, Theorem 9]. When $m=1$, it reduces to the absolute bound of Delsarte, Goethals and Seidel [12, Theorem 6.1]. There is also a relative bound, which depends on the actual values of the inner products and is sometimes tighter. The relative bound for real Grassmannian spaces was given by Bachoc [2, Proposition 2.3].

Theorem 5 Let $f\left(x_{1}, \ldots, x_{m}\right) \in \mathbb{R}\left[x_{1}, \ldots, x_{m}\right]$ be a symmetric polynomial such that $f=\sum_{\mu} c_{\mu} Z_{\mu}$, where $Z_{\mu}$ is a zonal orthogonal polynomial, and each $c_{\mu} \geq 0$. Further assume that $c_{(0)}$ is strictly positive. If $S$ is a set of subspaces in $\mathcal{G}_{m, n}$ such that $f_{a}(b):=f\left(y_{1}(a, b), \ldots, y_{m}(a, b)\right)$ is nonpositive for every $a \neq b$ in $S$, then

$$
|S| \leq \frac{f(1, \ldots, 1)}{c_{(0)}} .
$$

Proof Since $f_{a}(b) \leq 0$ for $b \neq a$, summing over all $b \in S$, we have

$$
\sum_{b \in S} f_{a}(b) \leq f_{a}(a)=f(1, \ldots, 1) .
$$

Then averaging over all $a \in S$,

$$
\begin{aligned}
f(1, \ldots, 1) & \geq \frac{1}{|S|} \sum_{a, b \in S} f_{a}(b) \\
& =\frac{1}{|S|} \sum_{\mu} c_{\mu} \sum_{a, b \in S} Z_{\mu}(a, b) .
\end{aligned}
$$

By Lemma 3, the inner sum is non-negative for $\mu \neq 0$. If $\mu=(0)$, then $Z_{(0)}(a, b)=1$ for all $a$ and $b$, and hence,

$$
\begin{aligned}
f(1, \ldots, 1) & \geq \frac{1}{|S|} c_{(0)} \sum_{a, b \in S} 1 \\
& =c_{(0)}|S| .
\end{aligned}
$$

Equality holds if and only if $f_{a}(b)=0$ for every $a \neq b \in S$ and for each $\mu \neq(0)$, we have either $c_{\mu}=0$ or $\sum_{a \in S} Z_{\mu, a}=0$. (We will see in Section 9 that when $c_{\mu}>0$ for all $|\mu| \leq \operatorname{deg}(f)$, this implies that we have a Grassmannian $t$-design.)

By way of example, we consider the case of a single nontrivial distance in detail. The following result is known as the complex Grassmannian simplex bound and can also be obtained from the real Grassmannian simplex bound by embedding $\mathbb{C}^{n}$ into $\mathbb{R}^{2 n}$ : see Corollary 4 in Section 7.

Corollary 2 Let $S$ be a subset of $\mathcal{G}_{m, n}$ such that $\operatorname{tr}\left(P_{a} P_{b}\right) \in[0, \alpha]$ for all $a \neq b$ in $S$, and $\alpha<m^{2} / n$. Then

$$
|S| \leq \frac{n(m-\alpha)}{m^{2}-n \alpha} .
$$


Proof The first two zonal orthogonal polynomials are $Z_{(0)}(y)=1$ and (up to normalization) $Z_{(1)}(y)=\sum_{i=1}^{m} y_{i}-m^{2} / n$. The annihilator for $\alpha$ is the polynomial

$$
f\left(y_{1}, \ldots, y_{m}\right)=\sum_{i=1}^{m} y_{i}-\alpha,
$$

and if $y_{1}, \ldots, y_{m}$ are the principal angles of $a$ and $b$ in $S$, then $f_{a}(b)=f(y(a, b))=0$. In terms of zonal polynomials, we have

$$
\begin{aligned}
f\left(y_{1}, \ldots, y_{m}\right) & =\sum_{i=1}^{m} y_{i}-\alpha \\
& =Z_{(1)}(y)+\left(\frac{m^{2}}{n}-\alpha\right) Z_{(0)}(y) .
\end{aligned}
$$

Applying Theorem 5, we get

$$
|S| \leq \frac{f(1, \ldots, 1)}{c_{(0)}}=\frac{m-\alpha}{m^{2} / n-\alpha} .
$$

In particular, if $S$ is a 1-distance set with non-trivial inner product $\alpha$, then Corollary 2 applies, and the bound is tighter than the bound in Corollary 1 provided that $\alpha<1 /(n+1)$. When $m=1$, Corollary 2 reduces to Delsarte, Goethals and Seidel's bound for a set of complex equiangular lines:

$$
|S| \leq \frac{n(1-\alpha)}{1-n \alpha} .
$$

Similarly, using the zonal orthogonal polynomials $Z_{(0)}, Z_{(1)}, Z_{(1,1)}$ and $Z_{(2)}$, we get a bound using the annihilator of two distances.

Corollary 3 Let $S$ be a subset of $\mathcal{G}_{m, n}$ such that $\operatorname{tr}\left(P_{a} P_{b}\right) \in[\alpha, \beta]$ for all $a \neq b$ in $S$. Further assume that

$$
\begin{array}{r}
\alpha+\beta \leq \frac{2\left(m^{2} n-4 m+n\right)}{n^{2}-4}, \\
\alpha+\beta-\frac{n \alpha \beta}{m^{2}}<\frac{m^{2} n-2 m+n}{n^{2}-1} .
\end{array}
$$

Then

$$
|S| \leq \frac{n(m-\alpha)(m-\beta)}{m^{2}\left[\frac{(m+1)^{2}}{2(n+1)}+\frac{(m-1)^{2}}{2(n-1)}-(\alpha+\beta)+\frac{n \alpha \beta}{m^{2}}\right]} .
$$

When $m=1$ this reduces to the Delsarte, Goethals and Seidel's bound of

$$
|S| \leq \frac{n(n+1)(1-\alpha)(1-\beta)}{2-(n+1)(\alpha+\beta)+n(n+1) \alpha \beta}
$$

for 2-distance sets of lines in complex projective space $\mathbb{C} P^{n-1}$. 
Proof The annihilator for $\{\alpha, \beta\}$ is

$$
f(y)=\left(\sum_{i=1}^{m} y_{i}-\alpha\right)\left(\sum_{i=1}^{m} y_{i}-\beta\right)
$$

and is nonpositive if $\langle a, b\rangle \in[\alpha, \beta]$. In terms of Schur polynomials, the annihilator is

$$
f(y)=\left(\begin{array}{c}
m+1 \\
2
\end{array}\right) X_{(2)}^{*}(y)+\left(\begin{array}{c}
m \\
2
\end{array}\right) X_{(1,1)}^{*}(y)-(\alpha+\beta) m X_{(1)}^{*}(y)+\alpha \beta .
$$

Writing each Schur polynomial in terms of zonal orthogonal polynomials, we get

$$
\begin{aligned}
f(y)= & c_{(2)} Z_{(2)}+c_{(1,1)} Z_{(1,1)}+c_{(1)} Z_{(1)}+\frac{m^{2}}{n}\left[\frac{(m+1)^{2}}{n+2}+\frac{(m-1)^{2}}{n-2}-(\alpha+\beta)\right] \\
& +\alpha \beta-\frac{m^{2}(m+1)^{2}}{2(n+1)(n+2)}-\frac{m^{2}(m-1)^{2}}{2(n-1)(n-2)},
\end{aligned}
$$

for some constants $c_{(2)}, c_{(1,1)}$, and $c_{(1)}$. The resulting bound $f(1,1, \ldots, 1) / c_{(0)}$ from Theorem 5 simplifies to

$$
\frac{n(m-\alpha)(m-\beta)}{m^{2}\left[\frac{(m+1)^{2}}{2(n+1)}+\frac{(m-1)^{2}}{2(n-1)}-(\alpha+\beta)+\frac{n \alpha \beta}{m^{2}}\right]} .
$$

Conditions (3) and (4) arise from insisting that $c_{(1)} \geq 0$ and $c_{(0)}>0$ respectively.

\section{Other bounds}

Certain cases of equality in Corollaries 2 and 3 also achieve equality for bounds on the size of the largest inner product that occurs in a set of subspaces. For real Grassmannians, Conway, Hardin and Sloane [9] call these bounds the simplex and orthoplex bounds. Here we give their complex analogues.

Recall that if $P_{a}$ is the $n \times n$ projection matrix for $a \in \mathcal{G}_{m, n}$, then $P_{a}$ is Hermitian with trace $m$, so $P_{a}^{\prime}=P_{a}-m I / n$ lies in a real space of dimension $n^{2}-1$. Moreover $\left\|P_{a}^{\prime}\right\|^{2}:=\operatorname{tr}\left(P_{a}^{\prime} P_{a}^{\prime}\right)=m(1-m / n)$, so $P_{a}^{\prime}$ is embedded onto a sphere of radius $\sqrt{m(1-m / n)}$ in $\mathbb{R}^{n^{2}-1}$. Further recall that the chordal distance on $\mathcal{G}_{m, n}$ is defined by

$$
\begin{aligned}
d_{c}(a, b)^{2} & =m-\operatorname{tr}\left(P_{a} P_{b}\right) \\
& =\frac{1}{2}\left\|P_{a}-P_{b}\right\|^{2}=\frac{1}{2}\left\|P_{a}^{\prime}-P_{b}^{\prime}\right\|^{2} .
\end{aligned}
$$

With this distance, the Grassmannians are isometrically embedded into $\mathbb{R}^{n^{2}-1}$. The "Rankin bounds" given in Theorem 6 below (see [5, Theorems 6.1.1 \& 6.1.2]) are bounds on the minimum distance between points on a real sphere as a function of the number of points and the dimension of the space. An equatorial simplex refers to a 
set of $N$ points on the unit sphere that form a simplex in a hyperplane of dimension $N-1$.

Theorem 6 Given $N$ points on a sphere of radius $r$ in $\mathbb{R}^{D}$, the minimum distance $d$ between any two points satisfies

$$
d \leq r \sqrt{\frac{2 N}{N-1}} .
$$

Equality requires $N \leq D+1$ and occurs if and only if the points form a regular equatorial simplex. For $N>D+1$, the minimum distance satisfies

$$
d \leq r \sqrt{2}
$$

and equality requires $N \leq 2 D$. When $N=2 D$, equality occurs if and only if the points are the vertices of a regular orthoplex.

Conway, Hardin and Sloane [9] apply these bounds to get the simplex and orthoplex bounds for real Grassmannians: we can do the same for the complex Grassmannians.

Corollary 4 Given a finite set $S \subseteq \mathcal{G}_{m, n}$, the largest inner product $\alpha=\langle a, b\rangle$ between any two subspaces in $S$ satisfies

$$
\alpha \geq m \frac{m|S|-n}{n|S|-n} .
$$

Equality requires $|S| \leq n^{2}$ and occurs if and only if the subspaces form a regular equatorial simplex in $\mathbb{R}^{n^{2}-1}$. For $|S|>n^{2}$, the largest inner product satisfies

$$
\alpha \geq \frac{m^{2}}{n},
$$

and equality requires $|S| \leq 2\left(n^{2}-1\right)$. Equality occurs if the subspaces form the $2\left(n^{2}-1\right)$ vertices of a regular orthoplex in $\mathbb{R}^{n^{2}-1}$.

If $S$ is an $\{\alpha\}$-code, then solving inequality (5) for $|S|$ recovers the relative bound in Corollary 2. Moreover, if $|S|=n^{2}$ (equality in the absolute bound of Corollary 1), then

$$
\alpha=\frac{m(m n-1)}{n^{2}-1} .
$$

On the other hand, if $S$ is a $\left\{0, m^{2} / n\right\}$-code, and $m=n / 2$, then the relative bound in Corollary 3 implies that

$$
|S| \leq 2\left(n^{2}-1\right)
$$

which corresponds to equality in the orthoplex bound (6). 


\section{Examples}

In this section we give examples demonstrating the tightness of the bounds in the previous sections.

When the rank $m$ of the Grassmannian subspaces is 1 , we recover all the classical results of Delsarte, Goethals and Seidel [12] for lines in complex projective space: their paper gives several examples of bounds with equality. In particular, the upper bound for $\{\alpha\}$-codes in $\mathbb{C} P^{n-1}$ is $n^{2}$, and equality can only hold with a trace inner product value of $\alpha=1 /(n+1)$. Examples of tightness have been found for several small values of $n$ and are conjectured to exist for every $n$. These equiangular lines are sometimes called symmetric informationally complete POVMs in the quantum information literature: see [24] for more details or [21] for recent results. Another important example in $\mathcal{G}_{1, n}$ is the relative bound (Corollary 3 ) with inner product values of $\alpha=0$ and $\beta=1 / n$. The upper bound for the size of a $\{0,1 / n\}$-code is $n(n+1)$, and when equality is achieved the code is known as a maximal set of mutually unbiased bases. Constructions achieving the bound are known when $n$ is a prime power; see [16] for some constructions and [25] for applications to quantum information.

In the case $m=n / 2$, if $a$ is in $\mathcal{G}_{m, n}$, then its orthogonal complement $a^{\perp}$ is also in $\mathcal{G}_{m, n}$, and $a$ and $a^{\perp}$ have a trace inner product of 0 . Here again, such subspaces have applications in quantum state tomography; more details will be found in [15]. If $S$ is a $\{0, n / 4\}$-code in $\mathcal{G}_{n / 2, n}$, then by the relative bound (Corollary 3 ), $S$ has size at most $2\left(n^{2}-1\right)$. In these case we may assume that both $a$ and $a^{\perp}$ are in $S$, because if $a$ and $b$ have a trace inner product of $n / 4$, then so do $a^{\perp}$ and $b$. The following construction, due to Martin Rötteler, is readily verified and demonstrates that Corollary 3 is tight when $n$ is a power of 2 .

Theorem 7 Let $X_{1}, \ldots, X_{n^{2}-1}$ be the Pauli matrices of order $n=2^{k}$, and let

$$
M_{i}:=\frac{1}{2}\left(I+X_{i}\right)
$$

Then $\bigcup_{i=1}^{n^{2}-1}\left\{M_{i}, I-M_{i}\right\}$ is the set of projection matrices for a $\{0, n / 4\}$-code of size $2\left(n^{2}-1\right)$ in $\mathcal{G}_{n / 2, n}$.

More generally, the bound is tight when $n$ is the order of a Hadamard matrix: details will appear in [15].

When the dimension of the complex space is an odd prime power, there is another construction which achieves the relative bound with equality. The following is the complex version of a set of real Grassmannian packings due to Calderbank, Hardin, Rains, Shor, and Sloane [8]. For lack of another reference in the complex case, the details are included here.

Let $V:=\mathbb{F}_{q}^{n}$, where $q=p^{k}$ and $p$ is an odd prime, and let $\left\{e_{v}: v \in V\right\}$ be the standard basis for $\mathbb{C}^{q^{n}}$. Then define the $q^{n} \times q^{n}$ Pauli matrices

$$
\begin{aligned}
& X(a): e_{v} \mapsto e_{v+a}, \\
& Y(a): e_{v} \mapsto \omega^{\operatorname{tr}\left(a^{T} v\right)} e_{v},
\end{aligned}
$$


where $\omega$ is a primitive $p$-th root of unity. Note that $e_{v}$ is an eigenvalue for $Y(a)$ and $e_{v}^{*}:=\sum_{a} \omega^{\operatorname{tr}\left(a^{T} v\right)} e_{a}$ is an eigenvalue for $X(a)$. Define the extraspecial Pauli group $E$ to be generated by all $X(a), Y(a)$, and $\omega I$; it has $p q^{n}$ elements, all of the form $\omega^{i} X(a) Y(b)$, for $i \in \mathbb{Z}_{p}, a, b \in V$. Its center is $Z(E)=\langle\omega I\rangle$, and $\bar{E}:=E / Z(E)$ is Abelian and therefore a vector space isomorphic to $V^{2}$ under the mapping

$$
(a, b) \mapsto X(a) Y(b) / Z(E) .
$$

The space $V^{2}$ has a nondegenerate alternating bilinear form (a symplectic form), namely

$$
\left\langle\left(a_{1}, b_{1}\right),\left(a_{2}, b_{2}\right)\right\rangle:=\operatorname{tr}\left(a_{1}^{T} b_{2}-a_{2}^{T} b_{1}\right) .
$$

It is not difficult to check that two elements in $E$, say $w^{i} X\left(a_{1}\right) Y\left(b_{1}\right)$ and $w^{j} X\left(a_{2}\right) Y\left(b_{2}\right)$, commute if and only if their images in $E / Z(E)$ satisfy

$$
\left\langle\left(a_{1}, b_{1}\right),\left(a_{2}, b_{2}\right)\right\rangle=0 .
$$

Subspaces on which the symplectic form vanishes are called totally isotropic. Therefore, a subspace $\bar{W}$ of $E / Z(E)$ is totally isotropic if and only if its preimage $W$ in $E$ is an Abelian subgroup.

We now use characters of subgroups of $E$ to define elements of $\mathcal{G}_{q^{k}}, q^{n}$. Let $\bar{W}$ be a totally isotropic subspace of $E / Z(E)$ of dimension $n-k$, and let $W$ be the preimage of $\bar{W}$ in $E$. If $\chi: \bar{W} \rightarrow \mathbb{C}$ is a character of $\bar{W}$, then $\chi^{\prime}: W \rightarrow \mathbb{C}$ defined by

$$
\chi^{\prime}\left(\omega^{i} X(a) Y(b)\right)=\omega^{-i} \chi(X(a) Y(b) / Z(E))
$$

is a character of $W$. Define a matrix

$$
\Pi_{\chi}:=\frac{1}{|W|} \sum_{g \in W} \chi^{\prime}(g) g .
$$

Lemma 6 If $\bar{W}$ is an $(n-k)$-dimensional totally isotropic subspace of $E / Z(E)$ and $\chi$ is a character of $\bar{W}$, then $\Pi_{\chi}$ is the projection matrix for a $q^{k}$-dimensional subspace of $\mathbb{C}^{q^{n}}$ which is invariant under the action of $W$.

Proof It is not difficult to check that $\Pi_{\chi}$ is Hermitian and $\Pi_{\chi}^{2}=\Pi_{\chi}$. It is also not difficult to check that $\Pi_{\chi} v$ is an eigenvector of $g \in W$ for any $v \in \mathbb{C}^{p^{n}}$, so $\Pi_{\chi}$ is a projection matrix for an invariant subspace. The rank of $\Pi_{\chi}$ is the trace of $\Pi_{\chi}$, which can be computed as follows, after noting that the only elements of $E$ with non-zero trace are the multiples of the identity:

$$
\operatorname{tr}\left(\Pi_{\chi}\right)=\frac{1}{|W|} \sum_{g=\omega^{i} I} \chi^{\prime}(g) \operatorname{tr}(g)=\frac{1}{p q^{n-k}} \sum_{i=1}^{p} \omega^{-i} \operatorname{tr}\left(\omega^{i} I\right)=q^{k} .
$$

In the construction that follows we require the $q$-binomial coefficients, defined as

$$
\left[\begin{array}{c}
n \\
m
\end{array}\right]_{q}:=\frac{\left(q^{n}-1\right) \ldots\left(q^{n-m+1}-1\right)}{\left(q^{m}-1\right) \ldots(q-1)}
$$


Theorem 8 For $0 \leq k \leq n-1$, let $S$ be the set of all $q^{k}$-dimensional invariant subspaces of the preimages $W$ of all $(n-k)$-dimensional totally isotropic subspaces $\bar{W}$ of $E / Z(E)$ (as described in Lemma 6$)$. Then $S$ is a $(n-k+1)$-distance set in $\mathcal{G}_{q^{k}, q^{n}}$ of size

$$
q^{n-k}\left[\begin{array}{c}
n \\
n-k
\end{array}\right]_{q} \prod_{i=k+1}^{n}\left(q^{i}+1\right) .
$$

Proof For $j \in\{1,2\}$, let $\bar{W}_{j}$ be an isotropic subspace of $E / Z(E)$, let $W_{j}$ be its Abelian preimage in $E$, let $\chi_{j}$ be a character of $\bar{W}_{j}$, and let $\Pi_{j}:=\Pi_{\chi_{j}}$ as in Lemma 6. Then

$$
\begin{aligned}
\operatorname{tr}\left(\Pi_{1} \Pi_{2}\right) & =\frac{1}{\left|W_{1}\right|\left|W_{2}\right|} \sum_{g_{1} \in W_{1}} \sum_{W_{2} \in S_{2}} \chi_{1}^{\prime}\left(g_{1}\right) \chi_{2}^{\prime}\left(g_{2}\right) \operatorname{tr}\left(g_{1} g_{2}\right) \\
& =\frac{1}{\left|W_{1}\right|\left|W_{2}\right|} \sum_{g_{1} \in W_{1} \cap W_{2}} \sum_{g_{2}=\omega^{i} g_{1}^{-1}} \chi_{1}^{\prime}\left(g_{1}\right) \chi_{2}^{\prime}\left(g_{2}\right) \operatorname{tr}\left(\omega^{i} I\right) \\
& =\frac{p q^{n}\left|W_{1} \cap W_{2}\right|}{\left|W_{1}\right|\left|W_{2}\right|}\left(\text { or } 0, \text { depending on } \chi_{1}^{\prime} \text { and } \chi_{2}^{\prime}\right) \\
& =\frac{q^{n} \mid \overline{W_{1} \cap W_{2} \mid}}{\left|\overline{W_{1}}\right|\left|\overline{W_{2}}\right|}(\text { or } 0) .
\end{aligned}
$$

Furthermore, any two distinct invariant subspaces from the same isotropic $\overline{W_{j}}$ are orthogonal. If $\overline{W_{1}} \neq \overline{W_{2}}$, then $\operatorname{dim}\left(\overline{W_{1} \cap W_{2}}\right) \in\{0,1, \ldots, n-k-1\}$ and so $\left|\overline{W_{1} \cap W_{2}}\right|$ takes $n-k$ possible values. It follows that $S$ is a $(n-k+1)$-distance set. To find the size of $S$, first note that the number of isotropic subspaces of dimension $n-k$ is (see [6, Lemma 9.4.1])

$$
\left[\begin{array}{c}
n \\
n-k
\end{array}\right]_{q} \prod_{i=k+1}^{n}\left(q^{i}+1\right)
$$

and then note that each isotropic subspace produces $q^{n-k}$ invariant subspaces.

In the case $k=n-1$, Theorem 8 produces a 2 -distance set in $\mathcal{G}_{q^{n-1}, q^{n}}$ of size $\frac{q\left(q^{2 n}-1\right)}{q-1}$. The inner product values that occur are $\alpha=0$ and $\beta=q^{n-2}$ : this construction achieves equality in the relative bound (Corollary 3). In his thesis, Zauner [30] has a construction which has these same parameters (in fact, Zauner's construction is more general, as it also allows $q$ to be an even prime power). In the case $k=n-2$, we get a 3-distance set in $\mathcal{G}_{q^{n-2}, q^{n}}$ of size $\frac{q^{2}\left(q^{2 n}-1\right)\left(q^{2 n-2}-1\right)}{\left(q^{2}-1\right)(q-1)}$, with inner product values $\alpha=0, \beta=q^{n-4}$, and $\gamma=q^{n-3}$.

There are many open questions regarding whether or not tightness in the bounds can be achieved; in particular, it is not known if there are any examples of subspaces achieving equality in the absolute bound (Corollary 1) for $m>1$. The smallest non- 
trivial case is a set of 16 subspaces of dimension 2 in $\mathbb{C}^{4}$, with an inner product value of $\alpha=14 / 15$.

\section{Designs}

In this section, we introduce the concept of a complex Grassmannian t-design. We give lower bounds for the size of a $t$-design and indicate the relationship between designs and codes.

Recall that $H_{t}(m, n)$ is the direct sum of the irreducible representations $H_{\mu}$ of $U(n)$ containing the zonal orthogonal polynomials $Z_{\mu, a}$, where $\mu$ is an integer partition of size at most $t$ and length at most $m . H_{t}(m, n)$ may also be thought of as the symmetric polynomials of degree at most $t$ in the principal angles of pairs of subspaces in $\mathcal{G}_{m, n}$.

We call a finite subset $S \subseteq \mathcal{G}_{m, n}$ a $t$-design if, for every polynomial $f$ in $H_{t}(m, n)$,

$$
\frac{1}{|S|} \sum_{a \in S} f(a)=\int_{\mathcal{G}_{m, n}} f(c) d c .
$$

In other words, the average of $f$ over $S$ is the same as the average of $f$ over the entire Grassmannian space. Recall that the average of $f$ over $\mathcal{G}_{m, n}$ can be written as $\langle 1, f\rangle$ : with this in mind we define an inner product for functions on $S$ as follows:

$$
\langle f, g\rangle_{S}:=\frac{1}{|S|} \sum_{a \in S} \overline{f(a)} g(a) .
$$

Then $S$ is a $t$-design if $\langle 1, f\rangle=\langle 1, f\rangle_{S}$ for every $f \in H_{t}(m, n)$. Equivalently, the zonal orthogonal polynomials $Z_{\mu, a}$ span $H_{\mu}$, so $S$ is a $t$-design if every $Z_{\mu, a}$ has the same averages over $S$ and over $\mathcal{G}_{m, n}$, where $\mu$ is a partition of at most $t$ into at most $m$ parts.

By way of example, consider Theorem 5. If $f=\sum_{\mu} c_{\mu} Z_{\mu}$ and $c_{\mu}>0$ for every $|\mu| \leq t$, then equality in Theorem 5 implies that $S$ is a $t$-design.

Before we give lower bounds for the size of a $t$-design, we offer two characterizations of designs.

Lemma 7 Let $S$ be a finite subset of $\mathcal{G}_{m, n}$. Then $S$ is a $t$-design if and only if for every $\mu$ such that $0<|\mu| \leq t$,

$$
\sum_{a, b \in S} Z_{\mu, a}(b)=0 .
$$

Proof Recall that $Z_{(0)}$ is the constant function, and every other $Z_{\mu}$ is orthogonal to $Z_{(0)}$, so

$$
\int_{\mathcal{G}_{m, n}} Z_{\mu, a}(b) d b=\left\langle 1, Z_{\mu, a}\right\rangle=0
$$


for every $a \in \mathcal{G}_{m, n}$ and $\mu \neq(0)$. Thus $S$ is a $t$-design if and only if

$$
\frac{1}{|S|} \sum_{b \in S} Z_{\mu, a}(b)=\frac{1}{|S|} \sum_{b \in S} Z_{\mu, b}(a)=0
$$

for all $a$ and $0<|\mu| \leq t$, which means that $\sum_{b \in S} Z_{\mu, b}$ is identically 0 . From Lemma 3, this occurs if and only if $\sum_{a, b \in S} Z_{\mu, a}(b)=0$.

In the following, $P_{a}$ denotes the projection matrix of $a \in \mathcal{G}_{m, n}$.

Lemma 8 Let $S$ be a finite subset of $\mathcal{G}_{m, n}$. Then $S$ is a $t$-design if and only if

$$
\frac{1}{|S|} \sum_{a \in S} P_{a}^{\otimes t}=\int_{\mathcal{G}_{m, n}} P_{a}^{\otimes t} d a
$$

Proof By Lemma 5, $H_{t}$ is the space of homogeneous polynomials of degree $t$ in the entries of $P_{a}$. Therefore, the averages over $S$ and $\mathcal{G}_{m, n}$ agree for every polynomial in $H_{t}$ if and only if the averages of the entries in the $t$-th tensor products of the projection matrices also agree.

For the purposes of quantum tomography applications, 1- and 2-designs play a special role (see [15], as well as [26]). In those cases, we can evaluate the integral $\int P_{a}^{\otimes t} d a$ more explicitly. Let $T$ denote the "swap" operator $T: u \otimes v \mapsto v \otimes u$, for $u, v \in \mathbb{C}^{n}$.

Corollary 5 Let $S$ be a finite subset of $\mathcal{G}_{m, n}$. Then $S$ is a 1-design if and only if

$$
\frac{1}{|S|} \sum_{a \in S} P_{a}=\frac{m}{n} I
$$

Moreover, $S$ is a 2-design if and only if

$$
\frac{1}{|S|} \sum_{a \in S} P_{a} \otimes P_{a}=\frac{m}{n\left(n^{2}-1\right)}[(n m-1) I+(n-m) T] .
$$

Proof It is not difficult to check that $\int P_{a} d a=(m / n) I$ (see for example equation (2.2) of [25]). To evaluate $\int P_{a} \otimes P_{a} d a$, write $P_{a}=\sum_{i=1}^{m} a_{i} a_{i}^{*}$ for some orthonormal basis $\left\{a_{i}\right\}_{i=1}^{m}$ of $a$ and then use Lemma 5.3 of [25].

We now consider bounds for $t$-designs. The following absolute bound is the complex analogue of [3, Theorem 8].

Lemma 9 If $S$ is a $t$-design, then

$$
|S| \geq \operatorname{dim}\left(H_{\lfloor t / 2\rfloor}(m, n)\right) .
$$


Proof Let $\left\{e_{1}, \ldots, e_{N}\right\}$ be an orthonormal basis for $H_{\lfloor t / 2\rfloor}$. It follows from the unique decomposition of $L^{2}\left(\mathcal{G}_{m, n}\right)$ (or from Lemma 5) that $\bar{e}_{i} e_{j}$ is in $H_{2\lfloor t / 2\rfloor}$ and therefore in $H_{t}$. If $S$ is a $t$-design, and $\overline{e_{i}} e_{j}$ is in $H_{t}$, then

$$
\left\langle e_{i}, e_{j}\right\rangle=\left\langle 1, \overline{e_{i}} e_{j}\right\rangle=\left\langle 1, \overline{e_{i}} e_{j}\right\rangle_{S}=\left\langle e_{i}, e_{j}\right\rangle_{S},
$$

whence it follows that $\left\{e_{1}, \ldots, e_{N}\right\}$ are orthogonal as functions on $S$ (a space of dimension $|S|$ ).

If equality holds, then the basis for $H_{t / 2}(m, n)$ is also a basis for the functions on $S$. There is also a relative bound.

Theorem 9 Let $f\left(x_{1}, \ldots, x_{m}\right) \in \mathbb{R}\left[x_{1}, \ldots, x_{m}\right]$ be a symmetric polynomial such that $f=\sum_{\mu} c_{\mu} Z_{\mu}$, where $Z_{\mu}$ is a zonal polynomial for the Grassmannian space, and $c_{(0)}>0$. Further suppose $S$ is a $t$-design such that $f_{a}(b)=f\left(y_{1}(a, b), \ldots, y_{m}(a, b)\right)$ is nonnegative for every $a \neq b$ in $S$, and $c_{\mu} \leq 0$ for every $|\mu|>t$. Then

$$
|S| \geq \frac{f(1, \ldots, 1)}{c_{(0)}} .
$$

Proof Let $f_{a}$ be the zonal polynomial of $f$ at $a$, so that $f_{a}(b) \geq 0$ for $b \neq a$. Summing over all $b \in S$,

$$
|S|\left\langle 1, f_{a}\right\rangle_{S} \geq f_{a}(a)=f(1, \ldots, 1) .
$$

Again averaging over all $a \in S$,

$$
\begin{aligned}
f(1, \ldots, 1) & \leq \sum_{a \in S}\left\langle 1, f_{a}\right\rangle_{S} \\
& =\sum_{a \in S} \sum_{\mu} c_{\mu}\left\langle 1, Z_{\mu, a}\right\rangle_{S} \\
& =\sum_{\mu} c_{\mu} \sum_{a \in S}\left\langle 1, Z_{\mu, a}\right\rangle_{S} .
\end{aligned}
$$

Since $S$ is a $t$-design, the inner sum is zero for $0<|\mu| \leq t$. For $|\mu|>t$, the inner sum is nonnegative (by Lemma 3 ) and $c_{\mu} \leq 0$. Therefore,

$$
\begin{aligned}
f(1, \ldots, 1) & \leq c_{(0)} \sum_{a \in S}\left\langle 1, Z_{0, a}\right\rangle_{S} \\
& =c_{(0)}|S| .
\end{aligned}
$$

If equality holds, then we have $f_{a}(b)=0$ for every $a \neq b$ in $S$. That is, $S$ is an $f$-code. Furthermore, for every $|\mu|>t$, we have either $c_{\mu}=0$ or $\sum_{a \in S} Z_{\mu, a}=0$.

As with spherical codes and designs, the case where $S$ is both an $f$-code and a $t$-design is of particular interest, as the size of the set can be determined exactly. Combining Theorems 5 and 9 gives the following. 
Theorem 10 Suppose $S$ is an $f$-code for $f=\sum_{\mu} c_{\mu} Z_{\mu}$, where $c_{\mu} \geq 0$, and $S$ is also a $t$-design for $t \geq \operatorname{deg}(f)$. Then

$$
|S|=\frac{f(1,1, \ldots, 1)}{c_{(0)}} .
$$

Consider the following polynomial in $H_{t}(m, n)$ :

$$
Z_{t}:=\sum_{\substack{|\mu| \leq t \\ \operatorname{len}(\mu) \leq m}} Z_{\mu}
$$

This polynomial satisfies $\left\langle Z_{t, a}, f\right\rangle=f(a)$ for every $f \in H_{t}(m, n)$. Taking $f=Z_{t}$ in Theorem 10, we get:

Corollary 6 If $S$ is a $Z_{t}$-code and a $2 t$-design, then

$$
|S|=\operatorname{dim}\left(H_{t}(m, n)\right) .
$$

Theorem 11 Any two of the following imply the third:

(i) $S$ is an $f$-code, where $\operatorname{deg}(f)=t$;

(ii) $S$ is a 2t-design;

(iii) $|S|=\operatorname{dim}\left(H_{t}(m, n)\right)$.

Proof Suppose $S$ is a $f$-code with $|S|=\operatorname{dim}\left(H_{t}\right)$. Since equality holds in Theorem 4, the polynomials $\left\{f_{a}: a \in S\right\}$ are a basis for $H_{t}$. However, we have

$$
\left\langle Z_{t, a}, f_{b}\right\rangle=f_{b}(a)= \begin{cases}0, & b \neq a \\ f(1,1, \ldots, 1), & b=a .\end{cases}
$$

Thus $\left\{Z_{t, a}\right\}$ is a dual basis for $H_{t}$ and each $Z_{t, a}$ is a multiple of $f_{a}$. Now consider the averages $\left\langle Z_{t, a}, f_{b}\right\rangle_{S}$ : since $f_{a}(b)=Z_{t, a}(b)=0$ for $b \neq a$, we get

$$
\left\langle Z_{t, a}, f_{b}\right\rangle_{S}= \begin{cases}0, & b \neq a \\ f(1,1, \ldots, 1), & b=a\end{cases}
$$

Thus we have

$$
\left\langle 1, Z_{t, a} f_{b}\right\rangle_{S}=\left\langle\overline{Z_{t, a}}, f_{b}\right\rangle_{S}=\left\langle\overline{Z_{t, a}}, f_{b}\right\rangle=\left\langle 1, Z_{t, a} f_{b}\right\rangle
$$

for the bases $\left\{Z_{t, a}\right\}$ and $\left\{f_{b}\right\}$. But the set $\left\{Z_{t, a} f_{b}\right\}$ spans $H_{2 t}(n)$, so $S$ is a $2 t$-design.

Conversely, suppose $S$ is a $2 t$-design with $|S|=\operatorname{dim}\left(H_{t}\right)$, and let $f$ annihilate of the set of principal angles of $S$, so $S$ is an $f$-code. Since $H_{t}$ spans the functions on $|S|$, each $f_{a}$ is in $H_{t}$ and is therefore a polynomial of degree $t$. Thus $f$ has degree $t$. 
The simplest case of Theorem 11 is when $t=1$ : in this case, $S$ is a 1-distance set and a 2-design of size $n^{2}$. Moreover, $S$ is a $Z_{1}$-code, and $Z_{1}$ is the annihilator of $\frac{m(m n-1)}{n^{2}-1}$. Thus the inner product between every two distinct subspaces is

$$
\alpha=\frac{m(m n-1)}{n^{2}-1}
$$

\section{Association schemes}

As Theorem 11 indicates, sets of Grassmannian subspaces which reach equality in the Delsarte bounds have a great deal of structure. In this section, we show that-much like spherical codes and spherical designs - these sets often give rise to an association scheme.

Let $S$ be an $f$-code with a finite number of distinct sets of principal angles $y=$ $\left(y_{1}, \ldots, y_{m}\right)$. Denote the set of $y$ 's that occur by $\mathcal{Y}$. (Here, we include the trivial principal angles $(1, \ldots, 1)$.) For each $y \in \mathcal{Y}$, define a $|S| \times|S|$ matrix as follows:

$$
A_{y}(a, b):= \begin{cases}1, & a, b \text { have principal angles } y \\ 0, & \text { otherwise. }\end{cases}
$$

Each $A_{y}$ is a symmetric $\{0,1\}$-matrix. Furthermore, each pair $(a, b)$ has some principal angle $y$, so $\sum_{y \in \mathcal{Y}} A_{y}=J$, where $J$ is the all-ones matrix. If $y_{0}:=(1, \ldots, 1)$ denotes the trivial principal angles, then $A_{0}:=A_{y_{0}}$ is the identity matrix. We call the $A_{y}$ matrices Schur idempotents, as they are idempotent under Schur multiplication, defined as follows:

$$
(A \circ B)_{i j}:=A_{i j} B_{i j} .
$$

Under certain conditions, these Schur idempotents form an association scheme.

For each integer partition $\mu$ and corresponding zonal polynomial $Z_{\mu}$, define an $|S| \times|S|$ matrix as follows:

$$
E_{\mu}(a, b):=\frac{1}{|S|} Z_{\mu}(a, b) .
$$

Each $E_{\mu}$ is also symmetric and in the span of $\left\{A_{y}\right\}_{y \in \mathcal{Y}}$ :

$$
E_{\mu}=\frac{1}{|S|} \sum_{y \in \mathcal{Y}} Z_{\mu}(y) A_{y} .
$$

In particular, $E_{(0)}$ is a scalar multiple of $J$. When $\left\{A_{y}\right\}_{y \in \mathcal{Y}}$ forms an association scheme, the matrices $E_{\mu}$ are the scheme's idempotents.

Lemma 10 If $S$ is a $2 t$-design, then $\left\{E_{\mu}\right\}_{|\mu| \leq t, \operatorname{len}(\mu) \leq m}$ are a set of orthogonal idempotents. 
Proof Let $\mu$ and $\lambda$ satisfy $|\mu|,|\lambda| \leq t$ and $\operatorname{len}(\mu), \operatorname{len}(\lambda) \leq m$. Then

$$
\begin{aligned}
\left(E_{\mu} E_{\lambda}\right)_{a, b} & =\frac{1}{|S|^{2}} \sum_{c \in S} Z_{\mu}(a, c) Z_{\lambda}(c, b) \\
& =\frac{1}{|S|}\left\langle Z_{\mu, a}, Z_{\lambda, b}\right\rangle_{S} .
\end{aligned}
$$

Since $Z_{\mu, a}$ and $Z_{\lambda, b}$ are in $H_{t}$, their product is in $H_{2 t}$. Now $S$ is a $2 t$-design, so the average of $Z_{\mu, a} Z_{\lambda, b}$ over $S$ is the same as the average over $\mathcal{G}_{m, n}$. But

$$
\left\langle Z_{\mu, a}, Z_{\lambda, b}\right\rangle=\delta_{\lambda, \mu} Z_{\mu}(a, b),
$$

and so $E_{\mu} E_{\lambda}=\delta_{\lambda, \mu} E_{\mu}$.

More generally, if $|\mu|=i$ and $|\lambda|=j$, and $S$ is a $(i+j)$-design, then $E_{\mu}$ and $E_{\lambda}$ are orthogonal.

Now suppose $S$ is a $2 t$-design. By the previous lemma $\left\{E_{\mu}\right\}_{|\mu| \leq t}$ are linearly independent, and clearly the matrices $\left\{A_{y}\right\}_{y \in \mathcal{Y}}$ are also linearly independent. If $|\mathcal{Y}|$ equals the number of partitions of at most $t$ (into at most $m$ parts), then the span of $\left\{A_{y}\right\}_{y \in \mathcal{Y}}$ and $\left\{E_{\mu}\right\}_{|\mu| \leq t}$ are the same. Since $\left\{E_{\mu}\right\}_{|\mu| \leq t}$ is closed under multiplication, so too is the span of $\left\{A_{y}\right\}_{y \in \mathcal{Y}}$, and so we have an association scheme.

Corollary 7 Let $S$ be a $2 t$-design in $\mathcal{G}_{m, n}$ with principal angles set $\mathcal{Y}$ (including $(1, \ldots, 1))$. If $|\mathcal{Y}|$ is equal to the total number of partitions of $0,1, \ldots, t$ into at most $m$ parts, then $\left\{A_{y}\right\}_{y \in \mathcal{Y}}$ is an association scheme.

Lemma 11 Let $S$ be a $2 t$-design in $\mathcal{G}_{m, n}$ with principal angle set $\mathcal{Y}$ such that $|\mathcal{Y}|$ is the total number of partitions of $0,1, \ldots, t$ into at most $m$ parts. Then $\left\{E_{\mu}\right\}_{|\mu| \leq t, \operatorname{len}(\mu) \leq m}$ are the idempotents of the scheme $\left\{A_{y}\right\}_{y \in \mathcal{Y}}$.

Proof Since $E_{\mu}=\frac{1}{|S|} \sum_{y \in \mathcal{Y}} Z_{\mu}(y) A_{y}$, we see that the matrix $\left[Z_{\mu}(y)\right]$ is the transition matrix between the two bases of the association scheme and is therefore invertible. It follows that for each $y_{i}$ in $\mathcal{Y}$, some linear combination of the rows $Z_{\mu}$ forms a homogeneous degree- $t$ polynomial $g_{i}$ such that $g_{i}\left(y_{j}\right)=\delta_{i j}$. (Conversely, if such $g_{i}$ polynomials exist, then $\left[Z_{\mu}(y)\right]$ is invertible.) Then

$$
\begin{aligned}
\left(A_{i} E_{\mu}\right)_{a, b} & =\frac{1}{|S|} \sum_{c: y(a, c)=y_{i}} Z_{\mu}(c, b) \\
& =\left\langle g_{i, a}, Z_{\mu, b}\right\rangle_{S}=\left\langle g_{i, a}, Z_{\mu, b}\right\rangle .
\end{aligned}
$$

Now write $g_{i}=\sum_{|\lambda| \leq t} c_{i, \lambda} Z_{\lambda}$, so that

$$
\left\langle g_{i, a}, Z_{\mu, b}\right\rangle=\sum_{|\lambda| \leq t} c_{i, \lambda}\left\langle Z_{\lambda, a}, Z_{\mu, b}\right\rangle=c_{i, \mu} Z_{\mu}(a, b) .
$$

Thus $A_{i} E_{\mu}=c_{i, \mu} E_{\mu}$ for some $c_{i, \mu}$. 
By way of example, let $t=1$, and suppose $S$ is a 2-design with only two sets of principal angles, including the trivial one. The number of partitions of at most 1 is also two $(\mu=(0)$ and $\mu=(1))$, so by Corollary 7 we have an association scheme. In this case the scheme is the trivial one, namely $\{I, J-I\}$.

As another example of an association scheme obtained from principal angles, consider the collection of subspaces in $\mathcal{G}_{n / 2, n}$ from Theorem 7 . This collection has four distinct sets of principal angles:

$$
\begin{aligned}
& y=(1, \ldots, 1) \quad(\text { trivial principal angles }), \\
& y=(0, \ldots, 0) \quad\left(\text { angles between } a \text { and } a^{\perp}\right), \\
& y=(\underbrace{1, \ldots, 1}_{n / 4}, \underbrace{0, \ldots, 0}_{n / 4}), \\
& y=\left(\frac{1}{2}, \ldots, \frac{1}{2}\right) .
\end{aligned}
$$

While $|\mathcal{Y}|=4$ is the number of partitions of at most $2(\mu=(0), \mu=(1), \mu=(1,1)$ and $\mu=(2)$ ), the hypotheses of Corollary 7 are not satisfied because the subspaces do not form a 4-design. Nevertheless, it is easy to verify computationally that this collection does give a 3 -class association scheme.

We may define a coarser set of relations on an $f$-code $S$ using the inner products values $\langle a, b\rangle=\operatorname{tr}\left(P_{a} P_{b}\right)$ instead of the principal angles $y(a, b)$. Let $\mathcal{A}$ denote the set of nontrivial inner product values that occur in $S$, so $S$ is an $\mathcal{A}$-code. For $\alpha \in \mathcal{A}$ let $A_{\alpha}^{\prime}$ be the $|S| \times|S|$ matrix defined as follows:

$$
A_{\alpha}^{\prime}(a, b):= \begin{cases}1, & \langle a, b\rangle=\alpha \\ 0, & \text { otherwise }\end{cases}
$$

Also define $A_{m}^{\prime}:=I$ for the identity relation. Clearly each $A_{\alpha}^{\prime}$ is in the span of $\left\{A_{y}\right.$ : $y \in \mathcal{Y}\}$; in fact

$$
A_{\alpha}^{\prime}=\sum_{y \in \mathcal{Y}: \sum y_{i}=\alpha} A_{y} .
$$

In particular, $A_{m}^{\prime}=A_{0}=I$, and if 0 is in $\mathcal{A}$, then $A_{0}^{\prime}=A_{(0, \ldots, 0)}$. As before, the matrices are Schur idempotents and sum to $J$. Next we need the corresponding idempotents. For each $i \in\{0, \ldots, t\}$, define $E_{i}^{\prime}$ as follows:

$$
E_{i}^{\prime}:=\sum_{|\mu|=i} E_{\mu}
$$

This implies that $E_{0}^{\prime}=J /|S|$ and $E_{i}^{\prime}(a, b)=\left(Z_{i}(a, b)-Z_{i-1}(a, b)\right) /|S|$ for $i>0$ (where $Z_{i}$ is as defined in equation (8)). As in Lemma 10, if $S$ is a $2 t$-design, then $\left\{E_{i}^{\prime}: i \leq t\right\}$ is a set of orthogonal idempotents, and if $S$ is a $(2 t-1)$-design, then $\left\{E_{i}^{\prime}: i \leq t\right\}$ are linearly independent. 
Clearly $E_{i}^{\prime}$ is in the span of $\left\{A_{y}: y \in \mathcal{Y}\right\}$, since each $E_{\mu}$ is in that span. But suppose $Z_{i}(y)$ is the annihilator polynomial of some $i$-distance set, so it is a function only of the sum of the principal angles $\sum_{j} y_{j}$ rather than a symmetric function in $\left(y_{1}, \ldots, y_{m}\right)$. Then, in fact $E_{i}^{\prime}$ is in the span of $\left\{A_{\alpha}^{\prime}: \alpha \in \mathcal{A}\right\}$. If $Z_{i}(y)$ is an annihilator for sufficiently many $i$, then $\left\{E_{i}^{\prime}: 0 \leq i \leq t\right\}$ and $\left\{A_{\alpha}^{\prime}: \alpha \in \mathcal{A} \cup\{m\}\right\}$ span the same set, and that set is closed under multiplication.

Corollary 8 Let $S$ be a $2 t$-design that is also an $\mathcal{A}$-code in $\mathcal{G}_{m, n}$. If $|\mathcal{A}| \leq t$, and $Z_{i}(y)$ is an annihilator polynomial for each $i \leq t$, then $\left\{A_{\alpha}^{\prime}: \alpha \in \mathcal{A} \cup\{m\}\right\}$ is an association scheme.

In fact, these hypotheses can be weakened.

Theorem 12 Let $S$ be a $(2 t-2)$-design that is also an $\mathcal{A}$-code in $\mathcal{G}_{m, n}$. If $|\mathcal{A}|=t$, and $Z_{i}(y)$ is an annihilator for each $0 \leq i \leq t-1$, then $\left\{A_{\alpha}^{\prime}: \alpha \in \mathcal{A} \cup\{m\}\right\}$ is an association scheme.

Proof Since $S$ is a $2(t-1)$-design, the idempotents $\left\{E_{i}^{\prime}: 0 \leq i \leq t-1\right\}$ are linearly independent. We claim that $I$ is also linearly independent from $\left\{E_{i}^{\prime}: 0 \leq i \leq t-1\right\}$. For, if $I=\sum_{i=0}^{t-1} c_{i} E_{i}^{\prime}$, then the off-diagonal entries of $I$ are functions of a polynomial of degree at most $t-1$ in $\sum_{j} y_{j}$, namely

$$
\frac{1}{|S|}\left(c_{0}+\sum_{i=1}^{t-1} c_{i}\left(Z_{i}(y)-Z_{i-1}(y)\right)\right) .
$$

But all off-diagonal entries are 0 , implying that the polynomial has $t$ roots in $\sum_{i} y_{i}$, a contradiction. So $\left\{E_{i}^{\prime}: 0 \leq i \leq t-1\right\} \cup\{I\}$ is linearly independent and therefore spans $\left\{A_{\alpha}^{\prime}: \alpha \in \mathcal{A} \cup\{m\}\right\}$. Since it is closed under multiplication, we have an association scheme.

By way of example, suppose $t=2$ in Theorem 12 . Note that $Z_{0}(y)$ and $Z_{1}(y)$ are always annihilators. It follows that if $S$ is a 2-design, and the inner product set $\mathcal{A}=\{\langle a, b\rangle: a \neq b \in S\}$ contains exactly two distinct values, then $\left\{A_{\alpha}^{\prime}: \alpha \in \mathcal{A} \cup\{m\}\right\}$ is a 2-class association scheme. The association scheme obtained in this way from the construction in Theorem 7 is the complete multipartite scheme.

Corollary 9 Let $S$ be a $(2 t-2)$-design and an $\mathcal{A}$-code in $\mathcal{G}_{m, n}$ such that $|\mathcal{A}|=t$ and $Z_{i}(y)$ is an annihilator for $i \leq t-1$. Then the idempotents of the scheme $\left\{A_{\alpha}^{\prime}: \alpha \in\right.$ $\mathcal{A} \cup\{m\}\}$ are $E_{0}^{\prime}, \ldots, E_{t-1}^{\prime}$, and $I-\sum_{i=0}^{t-1} E_{i}^{\prime}$.

Proof Let $f_{\alpha}$ denote the annihilator polynomial of $\mathcal{A} \backslash\{\alpha\}$, normalized so that $f_{\alpha}(y)=1$ when $\sum_{i} y_{i}=\alpha$. Then $f_{\alpha}$ is a polynomial of degree $t-1$ in $\sum_{i} y_{i}$, and 
the corresponding zonal polynomial $f_{\alpha, a}$ is in $H_{t-1}(n)$. Writing $g_{i}:=Z_{i}-Z_{i-1}=$ $\sum_{|\mu|=i} Z_{\mu}$, we have

$$
\begin{aligned}
\left(A_{\alpha}^{\prime} E_{i}^{\prime}\right)_{a, b} & =\frac{1}{|S|} \sum_{\langle a, c\rangle=\alpha} g_{i}(y(c, b)) \\
& =\left\langle f_{\alpha, a}, g_{i, b}\right\rangle_{S}-\frac{f_{\alpha}(1, \ldots, 1)}{|S|} g_{i}(y(a, b)) \\
& =\left\langle f_{\alpha, a}, g_{i, b}\right\rangle-\frac{f_{\alpha}(1, \ldots, 1)}{|S|} g_{i}(y(a, b)) .
\end{aligned}
$$

Now decomposing $f_{\alpha}$ into its degrees as $f_{\alpha}=\sum_{i} c_{\alpha, i} g_{i}$, we get

$$
\begin{aligned}
\left(A_{\alpha}^{\prime} E_{i}^{\prime}\right)_{a, b} & =c_{\alpha, i}\left\langle g_{i, a}, g_{i, b}\right\rangle-\frac{f_{\alpha}(1, \ldots, 1)}{|S|} g_{i}(y(a, b)) \\
& =c_{\alpha, i} g_{i}(y(a, b))-\frac{f_{\alpha}(1, \ldots, 1)}{|S|} g_{i}(y(a, b)) \\
& =\left(c_{\alpha, i}|S|-f_{\alpha}(1, \ldots, 1)\right)\left(E_{i}^{\prime}\right)_{a, b} .
\end{aligned}
$$

Thus $A_{\alpha}^{\prime} E_{i}^{\prime}=\lambda_{\alpha, i} E_{i}^{\prime}$ for some constant $\lambda_{\alpha, i}$.

\section{Weighted designs}

In this section, we introduce a weighted version of the Grassmannian $t$-design, which is easier to construct than the unweighted one. Let $S$ be a finite subset of $\mathcal{G}_{m, n}$ and let $w: S \rightarrow \mathbb{R}$ be a positive function such that $\sum_{a \in S} w(a)=1$. Then $(S, w)$ is called a weighted $t$-design if, for every polynomial $f$ in $H_{t}(m, n)$,

$$
\sum_{a \in S} w(a) f(a)=\int_{\mathcal{G}_{m, n}} f(a) d a .
$$

In other words, the weighted average of every degree- $t$ polynomial over $S$ is the same as its average over $\mathcal{G}_{m, n}$. Every (unweighted) $t$-design is a weighted $t$-design with the constant weight function $w(a):=1 /|S|$.

The absolute and relative lower bounds for the size of a $t$-design also apply to weighted $t$-designs. In particular, from Lemma 9, the size of a weighted $t$-design is at least $\operatorname{dim}\left(H_{\lfloor t / 2\rfloor}(m, n)\right)$. Moreover, a result of Levenshtein [22, Theorem 4.3] can be adapted to show that equality holds if and only if the design is unweighted.

The advantage that weighted designs hold over unweighted designs is that it is always also possible to construct a weighted $t$-design of reasonable size in $\mathcal{G}_{m, n}$, for every $t, m$, and $n$. The following result is based on a construction of Godsil [14, Theorem 3.2].

Theorem 13 There exists a weighted $t$-design $(S, w)$ in $\mathcal{G}_{m, n}$ such that

$$
|S| \leq \operatorname{dim}\left(H_{t}(m, n)\right) .
$$


Proof Let $u:=|\{\mu: 0<|\mu| \leq t\}|$. For any $a \in \mathcal{G}_{m, n}$, define a zonal function $F_{t, a}$ : $\mathcal{G}_{m, n} \rightarrow \mathbb{R}^{u}$ as follows:

$$
F_{t, a}(b):=\left(Z_{\mu, a}(b)\right)_{(\mu: 0<|\mu| \leq t)} .
$$

In other words, the entries of the vector $F_{t, a}(b)$ are the values of the zonal polynomials $Z_{\mu, a}(b)$ in $H_{t}(m, n)$. Now recall that for any $|\mu|>0$,

$$
\int_{\mathcal{G}_{m, n}} Z_{\mu, a}(b) d a=\left\langle Z_{\mu, b}, 1\right\rangle=0,
$$

since $Z_{\mu}$ is orthogonal to the constant function $Z_{(0)}$. So the average of $Z_{\mu, a}$ over all $a \in \mathcal{G}_{m, n}$ is 0 , which implies that

$$
\int_{\mathcal{G}_{m, n}} F_{t, a} d a=(0, \ldots, 0) .
$$

Thus, the zero function $0: \mathcal{G}_{m, n} \rightarrow \mathbb{R}^{u}$ is in the convex hull of $\left\{F_{t, a}: a \in \mathcal{G}_{m, n}\right\}$. Carathéodory's theorem implies there is a finite subset $S \subseteq \mathcal{G}_{m, n}$ for which 0 is also in the convex hull of $\left\{F_{t, a}: a \in S\right\}$. More precisely, there is a positive weighting $w: S \rightarrow \mathbb{R}$ such that $\sum_{a \in S} w(a)=1$ and

$$
\sum_{a \in S} w(a) F_{t, a}=0
$$

which in turn implies that

$$
\sum_{a \in S} w(a) Z_{\mu, a}=0
$$

for every $0<|\mu| \leq t$. Therefore $(S, w)$ is a weighted $t$-design, by the argument in Lemma 7. Carathéodory's theorem also states that $S$ can be chosen with cardinality at most the dimension of the span of $\left\{F_{t, a}: a \in \mathcal{G}_{m, n}\right\}$. Since the zonal polynomials span $H_{t}(m, n)$, the dimension of this space is $\operatorname{dim}\left(H_{t}(m, n)\right)$.

Acknowledgements The author would like to thank Martin Rötteler, Chris Godsil, Bill Martin, Barry Sanders, and an anonymous referee for their helpful comments. This work was funded by NSERC, MITACS, PIMS, iCORE, and the University of Calgary Department of Mathematics and Statistics Postdoctoral Program.

\section{References}

1. Agrawal, D., Richardson, T.J., Urbanke, R.L.: Multiple-antenna signal constellations for fading channels. IEEE Trans. Inf. Theory 47, 2618-2626 (2001)

2. Bachoc, C.: Linear programming bounds for codes in Grassmannian spaces. IEEE Trans. Inf. Theory 52, 2111-2125 (2006)

3. Bachoc, C., Bannai, E., Coulangeon, R.: Codes and designs in Grassmannian spaces. Discrete Math. 277, 15-28 (2004)

4. Bachoc, C., Coulangeon, R., Nebe, G.: Designs in Grassmannian spaces and lattices. J. Algebr. Comb. 16, 5-19 (2002) 
5. Böröczky Jr., K.: Finite Packing and Covering. Cambridge Tracts in Mathematics, vol. 154. Cambridge University Press, Cambridge (2004)

6. Brouwer, A.E., Cohen, A.M., Neumaier, A.: Distance-Regular Graphs. Springer, Berlin (1989)

7. Bump, D.: Lie Groups. Graduate Texts in Mathematics, vol. 225. Springer, New York (2004)

8. Calderbank, A.R., Hardin, R.H., Rains, E.M., Shor, P.W., Sloane, N.J.A.: A group-theoretic framework for the construction of packings in Grassmannian spaces. J. Algebr. Comb. 9, 129-140 (1999)

9. Conway, J.H., Hardin, R.H., Sloane, N.J.A.: Packing lines, planes, etc.: packings in Grassmannian spaces. Exp. Math. 5, 139-159 (1996)

10. Conway, J.H., Sloane, N.J.A.: Sphere Packings, Lattices and Groups, 2nd edn. Grundlehren der Mathematischen Wissenschaften [Fundamental Principles of Mathematical Sciences], vol. 290. Springer, New York (1993)

11. Delsarte, P.: An algebraic approach to the association schemes of coding theory. Philips Res. Rep. Suppl. (1973), vi+97

12. Delsarte, P., Goethals, J.M., Seidel, J.J.: Bounds for systems of lines, and Jacobi polynomials. Philips Res. Rep. (1975), pp. 91-105

13. Fulton, W., Harris, J.: Representation Theory. Springer, New York (1991)

14. Godsil, C.D.: Polynomial spaces. In: Proceedings of the Oberwolfach Meeting "Kombinatorik", vol. 73 (1986), pp. 71-88 (1989)

15. Godsil, C.D., Rötteler, M., Roy, A.: Mutually unbiased subspaces, in preparation

16. Godsil, C.D., Roy, A.: Mutually unbiased bases, equiangular lines, and spin models. Eur. J. Comb. 30, 246-262 (2009)

17. Goodman, R., Wallach, N.R.: Representations and Invariants of the Classical Groups. Encyclopedia of Mathematics and its Applications, vol. 68. Cambridge University Press, Cambridge (1998)

18. Helgason, S.: Groups and Geometric Analysis. Pure and Applied Mathematics, vol. 113. Academic Press, Orlando (1984)

19. Horn, R.A., Johnson, C.R.: Matrix Analysis. Cambridge University Press, Cambridge (1990)

20. James, A.T., Constantine, A.G.: Generalized Jacobi polynomials as spherical functions of the Grassmann manifold. Proc. Lond. Math. Soc. (3) 29, 174-192 (1974)

21. Khatirinejad, M.: On Weyl-Heisenberg orbits of equiangular lines. J. Algebr. Comb. 28, 333-349 (2008)

22. Levenshtein, V.: On designs in compact metric spaces and a universal bound on their size. Discrete Math. 192, 251-271 (1998)

23. Nielsen, M.A., Chuang, I.L.: Quantum Computation and Quantum Information. Cambridge University Press, Cambridge (2000)

24. Renes, J., Blume-Kohout, R., Scott, A.J., Caves, C.M.: Symmetric informationally complete quantum measurements. J. Math. Phys. 45, 2171 (2004)

25. Roy, A., Scott, A.J.: Weighted complex projective 2-designs from bases: optimal state determination by orthogonal measurements. J. Math. Phys. 48, 072110 (2007)

26. Scott, A.J.: Tight informationally complete quantum measurements. J. Phys. A 39, 13507-13530 (2006)

27. Sepanski, M.R.: Compact Lie Groups. Graduate Texts in Mathematics, vol. 235. Springer, New York (2007)

28. Stanley, R.P.: Enumerative Combinatorics, vol. 2. Cambridge Studies in Advanced Mathematics, vol. 62. Cambridge University Press, Cambridge (1999)

29. Wong, Y.-c.: Differential geometry of Grassmann manifolds. Proc. Nat. Acad. Sci. USA 57, 589-594 (1967)

30. Zauner, G.: Quantendesigns. Ph.D. thesis, University of Vienna (1999) 